\title{
Modeling and SINR Analysis of Dual Connectivity in Downlink Heterogeneous Cellular Networks
}

\author{
Xianling Wang ${ }^{1}$, Min Xiao ${ }^{1}$, Hongyi Zhang ${ }^{1}$ and Sida Song ${ }^{2}$ \\ ${ }^{1}$ Fujian Key Laboratory of Communication Network and Information Processing \\ Xiamen University of Technology, Xiamen 361024, China \\ [e-mail: wangxianling@xmut.edu.cn] \\ ${ }^{2}$ Huawei Technologies Co. Ltd., Beijing, P. R. China \\ *Corresponding author: Xianling Wang
}

Received October 28, 2016; revised May 9, 2017; accepted June 19, 2017;

published November 30, 2017

\begin{abstract}
Small cell deployment offers a low-cost solution for the boosted traffic demand in heterogeneous cellular networks (HCNs). Besides improved spatial spectrum efficiency and energy efficiency, future HCNs are also featured with the trend of network architecture convergence and feasibility for flexible mobile applications. To achieve these goals, dual connectivity (DC) is playing a more and more important role to support control/user-plane splitting, which enables maintaining fixed control channel connections for reliability. In this paper, we develop a tractable framework for the downlink SINR analysis of DC assisted HCN. Based on stochastic geometry model, the data-control joint coverage probabilities under multi-frequency and single-frequency tiering are derived, which involve quick integrals and admit simple closed-forms in special cases. Monte Carlo simulations confirm the accuracy of the expressions. It is observed that the increase in mobility robustness of DC is at the price of control channel SINR degradation. This degradation severely worsens the joint coverage performance under single-frequency tiering, proving multi-frequency tiering a more feasible networking scheme to utilize the advantage of DC effectively. Moreover, the joint coverage probability can be maximized by adjusting the density ratio of small cell and macro cell eNBs under multi-frequency tiering, though changing cell association bias has little impact on the level of the maximal coverage performance.
\end{abstract}

Keywords: Dual connectivity, heterogeneous cellular networks, Poisson point process, SINR analysis, stochastic geometry

A preliminary version of this paper appeared in WCSP 2016, October 13-15, Yangzhou, China. This version extends the preliminary work to the more complicated cross-tier interference scenario under single-frequency tiering and completes the derivation details for joint data-control channel SINR analysis. Besides, more extensive studies on the accuracy of the analytical expressions and the impacts of eNB densities, path loss exponents and cell association biases are performed in this version. This work was supported by the Natural Science Foundation of Fujian Province, China (NO. 2016J01323 and NO. 2017J01785). 


\section{Introduction}

$\mathbf{W}$ ith the rapid proliferation of smart devices, wireless cellular networks are experiencing an unprecedented growth in the amount of subscribers and data traffics. To meet this traffic demand, heterogeneity has been introduced into traditional single tier cellular networks. In the envisioned future heterogeneous cellular networks (HCNs), macro cell eNodeBs (eNBs), on which wide-area coverage is mostly relied, will be overlaying with dense low-power small cell eNBs. The network densification brings in a multitude of benefits to the existing network architectures. Firstly, by offloading traffics from macro cells to small cells and decreasing the distances between user equipments (UEs) and eNBs [1], the coverage performance and the spatial spectrum efficiency are improved. Secondly, exploiting cooperative interference avoidance mechanism [2], [3], the energy efficiency as a critical performance for future green communications can be optimized. Finally, through control/user-plane splitting [4] and network architectures convergence [5], [6], more flexible applications (e.g., data transmissions with high-speed mobility, machine type communications) for mobile users are allowed.

Since LTE Release 12, the mechanism of dual connectivity (DC) has been introduced as an important feature to support control/user-plane splitting in ultra-dense HCN [7]. In particular, DC mechanism enables a UE to be simultaneously associated with two different eNBs, the master eNB (MeNB) and secondary eNB (SeNB), which is a significant distinction from traditional single connectivity (SC). With DC mechanism applied in HCN, the macro cell eNBs and small cell eNBs can play the roles of the MeNBs and SeNBs, respectively. The feasibility of DC enhancement is on the base that the control signal and the data signal are physically separable, and that eNBs of different tiers can be connected via non-idea backhauls. By separately transmitting essential control-plane signals such as handover command from MeNBs, which usually cover larger areas, and transmitting data signals from SeNBs, which dramatically shorten communication distances, DC mechanism provides a flexible network architecture as an important role in the evolution of future ultra-dense HCN.

\subsection{Related Work}

To boost the standardization of DC mechanism in LTE, effort has been made in this field, which can be roughly divided into three categories. The work in the first category mainly applied DC to split traffics of a UE for data offloading and flow controlling. In [8], load balancing algorithm was applied and multiple associated small cells were simultaneously exploited for video transmission to improve the user satisfaction rate. In [9] and [10], two algorithms were proposed to optimize the traffic scheduling between MeNB and SeNB, bringing in effective increase in the network throughput. Similarly, in [11] and [12], inter-eNB flow control over non-ideal backhaul was performed and adjusted to generic 3GPP and realistic scenario to offer tradeoffs between MeNB and SeNB. In [13], the benefits of balancing load with DC was realized through cell association. In the second category, recent work addressed non-ideal backhaul constraint problem. The work in [14] and [15] proposed MeNB-SeNB grouping algorithms under non-ideal backhaul constraint to enhance both radio resource efficiency and backhaul capacity. Provided incomplete backhaul state information, the work in [16] and [17] addressed the flow bifurcation and the power allocation problem under DC mechanism. Note that above two categories of work mostly applied DC to offload data traffics, which was slightly different from the original purpose of DC mechanism to improve the mobility robustness. The work in the last category focused on evaluating the 
mobility robustness under DC mechanism. The work in [18] proposed a MRO scheme to improve mobility performance by adjusting the handover parameters to UE's velocity and other factors under DC mechanism. The authors in [19] and [20] assessed the time-of-stay and handover failure performance in generic 3GPP simulation, site-specific scenario and highway scenario, respectively, to investigate the mobility robustness of DC mechanism.

All these work have contributed to the standardization of DC mechanism in LTE, however, they mostly ignored the importance of signal-to-interference-plus-noise ratio (SINR) statistics in characterizing the physical layer network performance. Since the pioneer work in [21], cellular network SINR analysis with stochastic geometry tools has drawn so much attention. In [22], flexible cell association was taken into account and the impacts of density and cell association bias over outage probability and transmission rate of HCN were investigated. The work of [22] was extended in [23], which analytically captured the traffic load statistics. The flexibility and tractability of stochastic geometry tools also allow extending the analytical SINR evaluation to several comprehensive enhancements. The work in [24] considered device-to-device assisted HCN and applied stochastic geometry tools together with convex optimization to optimize the user density and transmit power. The authors in [25] further incorporated user behavior constraints in HCN and addressed the energy saving problem.

Despite the rich achievement for general HCN and some of its enhancement, blanks are still left unfilled so far in the SINR evaluation of DC assisted HCN. Considering that the utilization of DC mechanism may critically rely on the SINR performance, we are motivated to carry out the work in this paper.

\subsection{Contributions}

The objective of this paper is to propose an analytical framework for the joint data-control channel SINR characterization (i.e., joint coverage probability) in the HCN. We apply this framework to UE with and without DC capability in the downlink networks, which can be adopted as a flexible baseline model for further study. Specifically, the main contributions are summarized as follows:

- Tractable Model for Joint Data-Control Channel SINR Characterization: We develop to the best of our knowledge the first tractable framework to analyze the downlink joint data-control channel performance of HCN. Applying this framework, downlink joint coverage probability can be obtained through analytical expressions instead of time-consuming Monte Carlo simulations. The locations of macro cell eNBs and small cell eNBs are modeled as spatial Poisson point processes. To take both data and control channel into consideration, we define the data-control joint coverage probability, which is more adaptive to characterize the SINR performance of DC capable UEs. We carefully investigate the correlation between data and control channels from the aspects of desired signal and interference set. Based on these analyses, we provide general expressions of the data-control joint coverage probabilities for UEs with and without DC capability in both multi-frequency and single-frequency tiering scenarios.

- Dual Connectivity vs. Single Connectivity: We compare DC with traditional SC from the respect of joint coverage performance. We find that although DC provides a potential to improve the mobility robustness of HCN, it also brings in overall SINR degradation as a price. This degradation is mainly reflected on the control channel and is the consequence of fixedly receiving control signals from MeNB located further than the nearest SeNB.

- Multi-Frequency Tiering vs. Single-Frequency Tiering: We investigate the feasibility of DC in different network tiering schemes. Unsurprisingly, DC implementation proves to be frustrating in single-frequency tiering, where severe interference on the control 
channel can be expected. Whereas, multi-frequency tiering provides not only a more preferable joint coverage performance but also a chance to optimize the overall joint coverage performance by balancing the density ratio of SeNBs and MeNBs.

- Influences of Network Operation Parameters: We investigate the impacts of different network parameters to obtain design guidance for DC. We show that the joint coverage probability can be maximized by adjusting eNB density ratio. If UEs are encouraged to associate their data channel with SeNBs, lower density of SeNBs should be deployed to achieve the maximal joint coverage probability. However, once SINR targets are setup in a certain signal propagation scenario, the achievable maximum value will be fixed and changing association biases only alters the value of the optimal density ratio.

The rest of the paper is organized as follows. Section 2 gives a description of the downlink HCN model and the system setup, where DC mechanism is detailed from the aspects of interference and SINR. Section 3 starts the joint coverage evaluation from the multi-frequency tiering scenario. Section 4 extends the analysis to the more complicated single-frequency tiering which involves cross-tier interference. Given the derived expressions, numerical simulation results are discussed in Section 5. Finally, conclusions are drawn in Section 6.

\section{Network Model and DC Mechanism}

\subsection{Heterogeneous Cellular Network Model}

We consider a 2-tier HCN model comprising macro cell eNBs and small cell eNBs, which are independently distributed in the Euclidean plane to form two homogeneous Poisson point processes (PPPs) $\Phi_{1}$ and $\Phi_{2}$ with intensities $\lambda_{1}$ and $\lambda_{2}$ [26]. We use subscripts "1" and "2" to distinguish parameters of macro cell and micro cell, which are also referred to as the 1-st tier and the 2-nd tier. Previous work in [21] has discussed the accuracy of applying spatial point processes to model cellular networks and compared stochastic geometry approaches with system-level simulations. Denoting the location of the $i$-th eNB in $k$-th tier by $y_{i, k}$, we have $\Phi_{1}=\left\{y_{i, 1}, i \in \mathrm{N}\right\}$ and $\Phi_{2}=\left\{y_{i, 2}, i \in \mathrm{N}\right\}$. With a slight abuse of terminology, we also use $y_{i, k}$ to represent this eNB. UEs are uniformly distributed over the plane with high density which ensures all eNBs are active. An example of the network model is illustrated in Fig. 1.

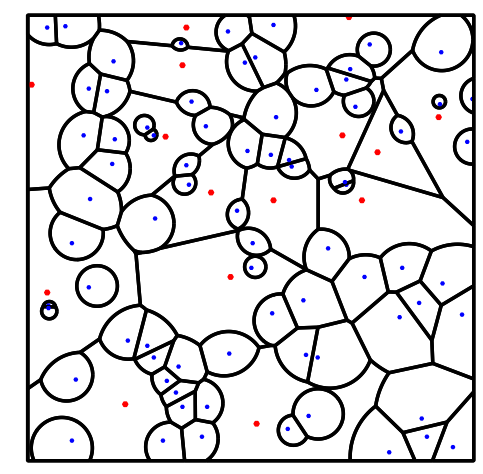

Fig. 1. An example of PPP based HCN model in a square area. The red markers are macro cell eNBs, while the denser blue markers are small cell eNBs. Weighted Voronoi tessellations are formed under the biased association criterion described in (1). 


\subsection{Joint Data-Control Signal Receiving Assumption and Channel Model}

We consider signal detections on both data channel and control channel. This is a significant distinction from previous work which focused on single data channel SINR characterization. In particular, a successful packet transmission requires correct signal demodulations on both data channel and control channel, which agrees with most wireless network systems. Take LTE as an example, in which data signal demodulation on Physical Downlink Shared Channel requires necessary control-plane information (e.g., modulation, coding scheme, scheduling indication) beared on Physical Downlink Control Channel. We also assume that data and control signals are on orthogonal time-frequency resources, which is common for OFDMA based systems.

As for channel model, the signal transmitted from eNB $y_{i, k}$ to a randomly chosen typical UE denoted as $\mathrm{UE}_{0}$ is attenuated by $\left\|y_{i, k}-x\right\|^{-\alpha_{k}}$ in large-scale, where $x$ is the location of $\mathrm{UE}_{0}$, $\left\|y_{i, k}-x\right\|$ represents the distance between $y_{i, k}$ and $x$, and $\alpha_{k}>2$ is the path loss exponent. For notational brevity, we let $\left\|y_{i, k}-x\right\|=Z_{i, k}$, and denote by $R_{k}$ the distance between $\mathrm{UE}_{0}$ and its nearest eNB in $k$-th tier. We also assume Rayleigh fast fading environment. Hence, the small-scale channel power gain $h_{i, k}$ and $g_{i, k}$ for data and control channels between $y_{i, k}$ and $\mathrm{UE}_{0}$ are both exponentially distributed with unit means. The eNBs in $k$-th tier transmit data and control signals with same power $P_{k}$. Therefore, the instantaneous received signal powers on data and control channel at $\mathrm{UE}_{0}$ from $y_{i, k}$ are given by $P_{k} h_{i, k} Z_{i, k}^{-\alpha_{k}}$ and $P_{k} g_{i, k} Z_{i, k}^{-\alpha_{k}}$, respectively.

\subsection{Cell Association and Dual Connectivity Mechanism}

For brevity, we distinguish UE with and without DC capability by prefix "DC-" and "SC-", respectively. With DC capability and that data and control channels are physically separable, DC-UE ${ }_{0}$ can manage its data signal and control signal from different tiers simultaneously.

For data channels, we consider biased cell association, where an UE is associated with the eNBs providing the strongest long-term averaged biased-received-power to receive data signals. All eNBs in $k$-th tier adopt identical positive bias factor $B_{k}$. Denoting the eNB managing $\mathrm{UE}_{0}$ 's data channel by $y_{m, n}$ of the $n$-th tier, we have

$$
y_{m, n}=\arg \max _{y_{i, k \in \emptyset D_{1} \cup \infty_{2}}} P_{k} B_{k} Z_{i, k}^{-\alpha_{k}} .
$$

Note that this biased cell association for data channel is applied for both DC-UE and SC-UE and makes $y_{m, n}$ the nearest eNB in the $n$-th tier. But with different bias factors between tiers, $y_{m, n}$ may not be the nearest eNB among all eNBs.

As for control channels, the control signals of DC-UE $\mathrm{U}_{0}$ are always managed by its nearest MeNB $y_{m, 1}$, while the control signals of SC-UE $E_{0}$ are managed by $y_{m, n}$ under (1), which can either be a MeNB or a SeNB and is the same as its data channel. We term MUE if UE receives data signals from MeNB, and SUE if UE receives data signals from SeNB. It should be aware that MUEs and SUEs are divided only according to data channel but not control channel.

Fig. 2 illustrates typical examples to understand the data/control signal splitting and distinguish $\mathrm{UE}_{0}$ with different connectivity modes. In all figures, coverage areas of MeNB and SeNB are divided under (1) and displayed in different background colors. Fig. 2(a) presents the serving links for a typical MUE. It shows that once located in the coverage area of MeNB, UEs with and without DC capability will both receive data and control signals from this 
MeNB. Fig. 2(b) and Fig. 2(c) reveal the major distinction between DC and SC. Although both located in the coverage area of SeNB, SC-SUE receives data and control signals from the same SeNB, while DC-SUE separately receives data signal from SeNB and control signal from MeNB. We also assume that MeNBs and SeNBs are connected through ideal backhaul links to share necessary signaling without any delay.

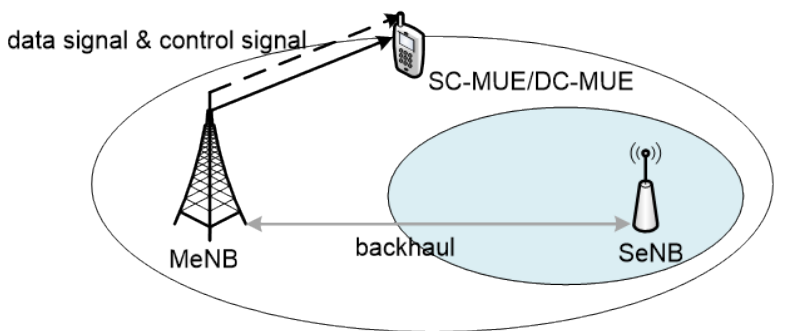

(a)

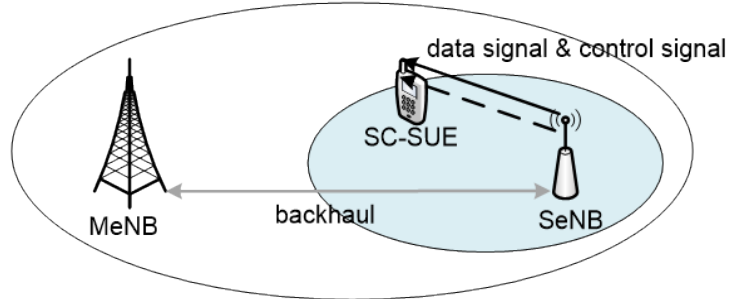

(b)

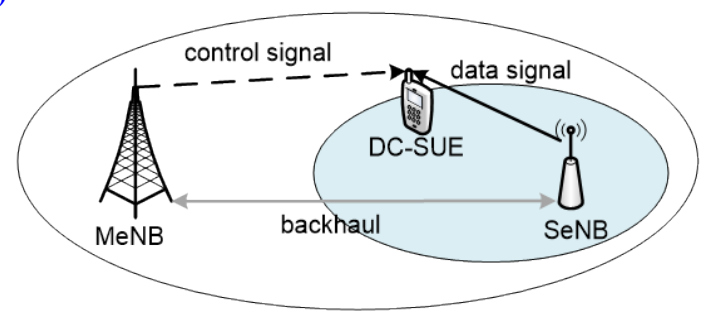

(c)

Fig. 2. Examples of typical HCN deployment with DC-UE and SC-UE.

\subsection{Interference and SINR Model}

We assume that frequency is fully reused for a tier resulting in co-tier interference between data channels within a tier, which is the same for control channels. However, data and control signals will not interfere with each other, since data and control channels are on orthogonal time-frequency resources.

As for cross-tier interference, we consider two general cases termed single-frequency tiering and multi-frequency tiering. For single-frequency tiering, macro cell tier and small cell tier share the same time-frequency resources. In consequence, data and control signals transmitted from MeNBs will interfere with those from SeNBs. For multi-frequency tiering, MeNBs and SeNBs will access different spectrums, so that both data and control channels will not suffer cross-tier interference. For now, multi-frequency tiering is highly advocated to effectively utilize the advantage of DC mechanism. So we mainly focus on DC assisted HCN under multi-frequency tiering. However, we will also analyze the single-frequency tiering case and investigate how the SINR performance will deteriorate in this case.

Based on the network model and assumptions described above, the SINR of the data signals, $\mathbf{S}_{\mathbf{D}}^{(\mathbf{M})}$ at SC-MUE $E_{0}$ and DC-MUE , and $\mathbf{S}_{\mathbf{D}}^{(\mathbf{S})}$ at SC-SUE $E_{0}$ and DC-SUE , are given by

$$
\mathrm{S}_{\mathrm{D}}^{(\mathrm{M})}=\frac{P_{1} h_{m, 1} R_{1}^{-\alpha_{1}}}{\sum_{y_{j, k} \in \Psi_{y_{m, 1}}} P_{k} h_{j, k} Z_{j, k}^{-\alpha_{k}}+\sigma^{2}},
$$

and

$$
\mathrm{S}_{\mathrm{D}}^{\mathrm{S})}=\frac{P_{2} h_{m, 2} R_{2}^{-\alpha_{2}}}{\sum_{y_{j, k} \in \Psi \backslash y_{m, 2}} P_{k} h_{j, k} Z_{j, k}^{-\alpha_{k}}+\sigma^{2}},
$$


where $P_{1}$ and $P_{2}$ are transmit powers of MeNB and SeNB, $\Psi \backslash y_{m, 1}$ represents excluding $y_{m, 1}$ from the set of $\Psi, R_{1}$ and $R_{2}$ are the distances from the nearest MeNB and SeNB to the UE, $h_{m, 1}$ and $h_{m, 2}$ are the small-scale channel power gains on the data channel from the nearest MeNB and SeNB to the UE, and $\sigma^{2}$ is the thermal noise power.

The SINR of the control signals $\mathrm{S}_{\mathrm{C}}^{(\mathrm{M})}$ at SC-MUE $\mathrm{S}_{0}$ and DC-MUE $\mathrm{E}_{0}$ are given by the same expression as

$$
\mathrm{S}_{\mathrm{C}}^{\mathrm{M})}=\frac{P_{1} g_{m, 1} R_{1}^{-\alpha_{1}}}{\sum_{y_{j, k} \in \Psi \Psi_{y_{m}, 1}} P_{k} g_{j, k} Z_{j, k}^{-\alpha_{k}}+\sigma^{2}},
$$

while the SINR of the control signals $\mathrm{S}_{\mathrm{C}}^{(\mathrm{S})}$ at $\mathrm{SC}-\mathrm{SUE}_{0}$ and DC-SUE $\mathrm{E}_{0}$ are given by different expressions as

$$
\mathrm{S}_{\mathrm{C}}^{(\mathrm{S})}= \begin{cases}\frac{P_{2} g_{m, 2} R_{2}^{-\alpha_{2}}}{\sum_{y_{j, k} \in \Psi y_{m, 2}} P_{k} g_{j, k} Z_{j, k}^{-\alpha_{k}}+\sigma^{2}}, & \text { for SC-SUE } \\ \frac{P_{1} g_{m, 1} R_{1}^{-\alpha_{1}}}{\sum_{y_{j, k} \in \Psi y_{y_{m, 1}} P_{k} g_{j, k} Z_{j, k}^{-\alpha_{k}}+\sigma^{2}}}, & \text { for DC-SUE }\end{cases}
$$

where $g_{m, 1}$ and $g_{m, 2}$ are the small-scale channel power gains on the control channel from the nearest MeNB and SeNB to the UE, respectively.

It should be noted that the interference sets $\Psi$ in (2) - (5) are the same for single-frequency tiering, while $\Psi$ is replaced by different PPP sets for multi-frequency tiering. Table 1 concludes the replacement of $\Psi$ according to different tiering cases, connectivity capabilities, and cell associations. Two significant features for the SINR characterization of DC are revealed in above SINR expressions and Table 1 as well.

1) The desired signal: Although the data channels of DC-SUE and SC-SUE share the same transmission distance expression $R_{2}$ in (3), the control signals of DC-SUE and SC-SUE experience different transmission distance expressions $R_{1}$ and $R_{2}$ in (5).

2) The interference set: Under multi-frequency tiering cases shown in Table 1, although the eNBs interfering the data signals of DC-SUE and SC-SUE form a same set $\Psi=\Phi_{2}$, the control signals of DC-SUE and SC-SUE suffer from different interference sets $\Psi=\Phi_{1}$ and $\Psi=\Phi_{2}$.

Above two features for SUEs are both due to the DC mechanism of separately managing data and control channel from different tiers described in Subsection 2.3. For MUEs, whether DC mechanism is supported or not, the desired data and control signals will always share the same transmission distance expression and suffer from the same interference sets.

Table 1. Realization of $\Psi$ under Different Cases

\begin{tabular}{|c|c|c|c|c|}
\hline & \multicolumn{2}{|c|}{ Data Channel } & \multicolumn{2}{c|}{ Control Channel } \\
\cline { 2 - 2 } & $\begin{array}{c}\text { Multi-Frequency } \\
\text { Tiering }\end{array}$ & $\begin{array}{c}\text { Single-Frequency } \\
\text { Tiering }\end{array}$ & $\begin{array}{c}\text { Multi-Frequency } \\
\text { Tiering }\end{array}$ & $\begin{array}{c}\text { Single-Frequency } \\
\text { Tiering }\end{array}$ \\
\hline \hline SC-MUE & $\Phi_{1}$ & & $\Phi_{1}$ & \multirow{2}{*}{$\Phi_{1}+\Phi_{2}$} \\
\hline DC-MUE & \multirow{2}{*}{$\Phi_{2}$} & $\Phi_{1}+\Phi_{2}$ & $\Phi_{2}$ & \\
\cline { 1 - 1 } SC-SUE & & & $\Phi_{1}$ & \\
\cline { 1 - 1 } DC-SUE & & &
\end{tabular}


To successfully demodulate signals on the data channel and control channel, $S_{D}$ and $S_{C}$ should be greater than specific SINR thresholds $T_{\mathrm{D}}$ and $T_{\mathrm{C}}$, respectively.

The main notations are summarized in Table 2.

Table 2. Notation Summary

\begin{tabular}{|c|c|}
\hline Notation & Description \\
\hline$\Phi_{k}, \lambda_{k}$ & PPP of eNBs in $k$-th tier and its density \\
\hline$y_{i, k}, x$ & Location of $i$-th eNB in $k$-th tier and typical UE \\
\hline$\alpha_{k}$ & Path loss exponent of $k$-th tier \\
\hline$Z_{i, k}$ & Distance between typical UE and $i$-th eNB in $k$-th tier \\
\hline$R_{k}$ & Distance between typical UE and its nearest eNB in $k$-th tier \\
\hline$h_{i, k}, g_{i, k}$ & $\begin{array}{l}\text { Small-scale channel power gain for data and control channel between typical UE and } \\
\qquad i \text {-th eNB in } k \text {-th tier }\end{array}$ \\
\hline$P_{k}$ & Transmit power for both data and control channel in $k$-th tier \\
\hline$B_{k}$ & Cell association bias of $k$-th tier \\
\hline$m, n$ & Indexes of eNB and tier managing typical UE's data channel \\
\hline$\sigma^{2}$ & Thermal noise power \\
\hline$\Psi$ & General interference set \\
\hline DC-SUE & DC capable UE with data and control channel managed by SeNB and MeNB \\
\hline DC-MUE & DC capable UE with data and control channel both managed by MeNB \\
\hline SC-SUE & DC incapable UE with data and control channel both managed by SeNB \\
\hline SC-MUE & DC incapable UE with data and control channel both managed by MeNB \\
\hline $\mathrm{S}_{\mathrm{D}}^{(\mathrm{M})}, \mathrm{S}_{\mathrm{D}}^{(\mathrm{S})}$ & SINR of data channel for typical MUE and typical SUE \\
\hline $\mathrm{S}_{\mathrm{C}}^{(\mathrm{M})}, \mathrm{S}_{\mathrm{C}}^{(\mathrm{S})}$ & SINR of control channel for typical MUE and typical SUE \\
\hline$T_{\mathrm{D}}, T_{\mathrm{C}}$ & SINR threshold for data and control channel to correctly demodulate signals \\
\hline $\mathrm{P}_{\mathrm{C}}$ & Data-control joint coverage probability \\
\hline $\mathrm{P}_{\mathrm{C} M \mathrm{M}}^{(\mathrm{SC})}, \mathrm{P}_{\mathrm{C} \mathrm{M}}^{(\mathrm{DCC})}$ & Conditional joint coverage probability for SC-MUE and DC-MUE \\
\hline $\mathrm{P}_{\mathrm{CS}}^{(\mathrm{SC})}, \mathrm{P}_{\mathrm{CS}}^{(\mathrm{DC})}$ & Conditional joint coverage probability for SC-SUE and DC-SUE \\
\hline $\mathrm{A}_{n}$ & Tier association probability that UE receives data signal from $n$-th tier \\
\hline
\end{tabular}

\section{SINR Performance Analysis under Multi-Frequency Tiering}

We focus on the downlink evaluation of DC assisted HCN and investigate the SINR performance from the respect of data-control joint coverage probability. We will firstly analyze this metric under multi-frequency tiering scenario in this section and leave the more complicated single-frequency tiering case to next section. The data-control joint coverage probability $\mathrm{P}_{\mathrm{C}}$ is defined as the probability that both data channel and control channel are covered with a sufficient SINR higher than thresholds $T_{\mathrm{D}}$ and $T_{\mathrm{C}}$, and is given by

$$
\mathrm{P}_{\mathrm{C}}=\mathrm{P}\left(\mathrm{S}_{\mathrm{D}} \geq T_{\mathrm{D}}, \mathrm{S}_{\mathrm{C}} \geq T_{\mathrm{C}}\right) .
$$

To obtain this metric, it is necessary to derive the tier association probabilities and the conditional data-control joint coverage probabilities. Therefore, we carry out the analysis over these metrics in the following subsections for a typical $\mathrm{UE}_{0}$, and this does not lose generality due to the stationarity of the PPP. 
It should be aware that $S_{D}$ and $S_{C}$ are highly correlated in large scale as revealed in (2) - (5). Take MUE as an example, which shows in (2) and (4) that the data and control channel share the same transmission distance and suffer the same interference set. For SUE, correlation can also be found with a small difference between DC-SUE and SC-SUE, which we will discuss later in Subsection 3.2. With this correlation, deviation can be expected if $P_{C}$ is obtained via multiplying $\mathrm{P}\left(\mathrm{S}_{\mathrm{D}} \geq T_{\mathrm{D}}\right)$ by $\mathrm{P}\left(\mathrm{S}_{\mathrm{C}} \geq T_{\mathrm{C}}\right)$ directly.

\subsection{Tier Association Probabilities}

As illustrated in Fig. 2, the serving links of MUEs and SUEs are quite different, especially when UEs are with DC capability. Due to this distinction, we firstly give the tier association probability $\mathrm{A}_{n}$ defined as the probability that $\mathrm{UE}_{0}$ receives data signals from the $n$-th tier. The basic idea to obtain $\mathrm{A}_{n}$ is to calculate the probability of event $P_{n} B_{n} R_{n}^{-\alpha_{n}} \geq \max _{k, k \neq n} P_{k} B_{k} R_{k}^{-\alpha_{k}}$, which is equivalently to $\prod_{k, k \neq n} \mathrm{P}\left(P_{n} B_{n} R_{n}^{-\alpha_{n}} \geq P_{k} B_{k} R_{k}^{-\alpha_{k}}\right)$. The result has been derived in Lemma 1 of [22], and is given by

$$
\mathrm{A}_{n}=2 \pi \lambda_{n} \int_{0}^{\infty} r \exp \left(-\pi \sum_{k=1}^{2} \lambda_{k}\left(\frac{P_{k} B_{k}}{P_{n} B_{n}}\right)^{\frac{2}{\alpha_{k}}} r^{\frac{2 \alpha_{n}}{\alpha_{k}}}\right) \mathrm{d} r .
$$

Equqtion (7) holds for both DC-UE and SC-UE since $A_{n}$ only involves data channel, which is treated in the same way for DC-UE and SC-UE.

\subsection{Conditional Joint Coverage Probabilities under Multi-Frequency Tiering}

To obtain the data-control joint coverage probabilities, we need to derive the conditional joint coverage probabilities of UEs receiving from different tiers. Denote by $\mathrm{P}_{\mathrm{C} \mid \mathrm{M}}^{(\mathrm{SC})}$ and $\mathrm{P}_{\mathrm{C} \mid \mathrm{M}}^{(\mathrm{DC})}$ the conditional joint coverage probabilities of SC-MUE $\mathrm{M}_{0}$ and DC-MUE $\mathrm{E}_{0}$, respectively. So we have $\mathrm{P}_{\mathrm{C} \mid \mathrm{M}}^{(\mathrm{SC})}=\mathrm{P}\left(\mathrm{S}_{\mathrm{D}}^{(\mathrm{M})} \geq T_{\mathrm{D}}, \mathrm{S}_{\mathrm{C}}^{(\mathrm{M})} \geq T_{\mathrm{C}} \mid n=1\right)$ and $\mathrm{P}_{\mathrm{C} \mid \mathrm{M}}^{(\mathrm{DC})}=\mathrm{P}\left(\mathrm{S}_{\mathrm{D}}^{(\mathrm{M})} \geq T_{\mathrm{D}}, \mathrm{S}_{\mathrm{C}}^{(\mathrm{M})} \geq T_{\mathrm{C}} \mid n=1\right)$. The following Lemma 1 provides the expressions of $\mathrm{P}_{\mathrm{C} \mid \mathrm{M}}^{(\mathrm{SC})}$ and $\mathrm{P}_{\mathrm{C} \mid \mathrm{M}}^{(\mathrm{DC})}$.

Lemma 1. In HCN, the conditional joint coverage probabilities of a typical SC-UE and a DC-UE receiving data signals from MeNBs under multi-frequency tiering are given by

$$
\begin{aligned}
\mathrm{P}_{\mathrm{C} \mid \mathrm{M}}^{(\mathrm{DC})}=\mathrm{P}_{\mathrm{C} \mid \mathrm{M}}^{(\mathrm{SC})}= & \frac{2 \pi \lambda_{1}}{\mathrm{~A}_{1}} \int_{0}^{\infty} r \exp \left(-\pi \lambda_{1} r^{2}\left(\mathrm{G}\left(T_{\mathrm{C}}, \alpha_{1}, 1\right)+\mathrm{G}\left(T_{\mathrm{D}}, \alpha_{1}, 1\right)\right)\right) \\
& \times \exp \left(-\frac{r^{\alpha_{1}} \sigma^{2}\left(T_{\mathrm{D}}+T_{\mathrm{C}}\right)}{P_{1}}-\pi \sum_{k=1}^{2} \lambda_{k}\left(\frac{P_{k} B_{k}}{P_{1} B_{1}}\right)^{\frac{2}{\alpha_{k}}} r^{\frac{2 \alpha_{1}}{\alpha_{k}}}\right) \mathrm{d} r,
\end{aligned}
$$

where $\mathrm{A}_{1}$ is given in (7), $\mathrm{G}(t, a, b)=\frac{2 t b^{\frac{2}{a}-1}}{a-2}{ }_{2} F_{1}\left(1,1-\frac{2}{a} ; 2-\frac{2}{a} ;-\frac{t}{b}\right)$, and ${ }_{2} F_{1}(\cdot)$ represents the Gauss hypergeometric function.

Proof: Conditioning on SC-UE receiving data signals from MeNB, $\mathrm{P}_{\mathrm{ClM}}^{(\mathrm{SC})}$ for $\mathrm{SC}-\mathrm{MUE}_{0}$ is given by 


$$
\mathrm{P}_{\mathrm{C} \mid \mathrm{M}}^{(\mathrm{SC})}=\mathrm{P}\left(\mathrm{S}_{\mathrm{D}}^{(\mathrm{M})} \geq T_{\mathrm{D}}, \mathrm{S}_{\mathrm{C}}^{(\mathrm{M})} \geq T_{\mathrm{C}} \mid n=1\right)=\frac{\mathrm{P}\left(\mathrm{S}_{\mathrm{D}}^{(\mathrm{M})} \geq T_{\mathrm{D}}, \mathrm{S}_{\mathrm{C}}^{(\mathrm{M})} \geq T_{\mathrm{C}}, n=1\right)}{\mathrm{P}(n=1)},
$$

where $\mathrm{P}(n=1)=\mathrm{A}_{1}$, and event " $n=1$ ” is equivalent to " $P_{1} B_{1} R_{1}^{-\alpha_{1}}>P_{2} B_{2} R_{2}^{-\alpha_{2}}$ ”. Though $\mathrm{S}_{\mathrm{D}}^{(\mathrm{M})}$ and $\mathrm{S}_{\mathrm{C}}^{(\mathrm{M})}$ share the same large-scale signal attenuation $R_{1}^{-\alpha_{1}}$ as mentioned previously, the channel power fluctuation $h_{m, 1}$ and $g_{m, 1}$ are independent in small-scale. Averaging over $R_{1}$ and $R_{2}$, the numerator of (9) can be derived as

$$
\begin{aligned}
& \mathrm{P}\left(\mathrm{S}_{\mathrm{D}}^{(\mathrm{M})} \geq T_{\mathrm{D}}, \mathrm{S}_{\mathrm{C}}^{(\mathrm{M})} \geq T_{\mathrm{C}}, n=1\right) \\
& =\int_{0}^{\infty} \mathrm{P}\left(\mathrm{S}_{\mathrm{D}}^{(\mathrm{M})} \geq T_{\mathrm{D}}, \mathrm{S}_{\mathrm{C}}^{(\mathrm{M})} \geq T_{\mathrm{C}}, R_{2}>\left(\frac{P_{2} B_{2}}{P_{1} B_{1}}\right)^{\frac{1}{\alpha_{2}}} r_{1}^{\frac{\alpha_{1}}{\alpha_{2}}}\right) \mathrm{f}_{R_{1}}\left(r_{1}\right) \mathrm{d} r_{1} \\
& \stackrel{(a)}{=} 4 \pi^{2} \lambda_{1} \lambda_{2} \int_{0}^{\infty} \int_{\left(\frac{P_{2} B_{2}}{A_{1} B_{1}}\right)^{\frac{1}{\alpha_{2}}} r_{1}^{\alpha_{1}}}^{\infty} \mathrm{P}\left(\mathrm{S}_{\mathrm{D}}^{(\mathrm{M})} \geq T_{\mathrm{D}}, \mathrm{S}_{\mathrm{C}}^{(\mathrm{M})} \geq T_{\mathrm{C}}\right) \cdot r_{2} \exp \left(-\pi \lambda_{2} r_{2}^{2}\right) \mathrm{d} r_{2} \cdot r_{1} \exp \left(-\pi \lambda_{1} r_{1}^{2}\right) \mathrm{d} r_{1} \\
& =2 \pi \lambda_{1} \int_{0}^{\infty} \mathrm{P}\left(\mathrm{S}_{\mathrm{D}}^{\mathrm{M})} \geq T_{\mathrm{D}}, \mathrm{S}_{\mathrm{C}}^{\mathrm{M})} \geq T_{\mathrm{C}}\right) r_{1} \exp \left(-\pi \lambda_{2}\left(\frac{P_{2} B_{2}}{P_{1} B_{1}}\right)^{\frac{2}{\alpha_{2}}} r_{1}^{\frac{2 \alpha_{1}}{\alpha_{2}}}-\pi \lambda_{1} r_{1}^{2}\right) \mathrm{d} r_{1} \\
& \stackrel{(b)}{\approx} 2 \pi \lambda_{1} \int_{0}^{\infty} \mathrm{P}\left(h_{m, 1} \geq \frac{T_{\mathrm{D}} r_{1}^{\alpha_{1}}}{P_{1}}\left(\sum_{\Psi \backslash y_{m .1}} P_{k} h_{j, k} Z_{j, k}^{-\alpha_{k}}+\sigma^{2}\right)\right) \mathrm{P}\left(g_{m, 1} \geq \frac{T_{\mathrm{C}} r_{1}^{\alpha_{1}}}{P_{1}}\left(\sum_{\Psi \backslash y_{m .1}} P_{k} h_{j, k} Z_{j, k}^{-\alpha_{k}}+\sigma^{2}\right)\right) \\
& \times r_{1} \exp \left(-\pi \sum_{k=1}^{2} \lambda_{k}\left(\frac{P_{k} B_{k}}{P_{1} B_{1}}\right)^{\frac{2}{\alpha_{k}}} r_{1}^{\frac{2 \alpha_{1}}{\alpha_{k}}}\right) \mathrm{d} r_{1} \\
& \stackrel{(c)}{=} 2 \pi \lambda_{1} \int_{0}^{\infty} \mathrm{L}_{\Phi_{1}}\left(\frac{T_{\mathrm{D}} r_{1}^{\alpha_{1}}}{P_{1}}\right) \mathrm{L}_{\Phi_{1}}\left(\frac{T_{\mathrm{C}} r_{1}^{\alpha_{1}}}{P_{1}}\right) r_{1} \exp \left(-\frac{r_{1}^{\alpha_{1}} \sigma^{2}\left(T_{\mathrm{D}}+T_{\mathrm{C}}\right)}{P_{1}}-\pi \sum_{k=1}^{2} \lambda_{k}\left(\frac{P_{k} B_{k}}{P_{1} B_{1}}\right)^{\frac{2}{\alpha_{k}}} r_{1}^{\frac{2 \alpha_{1}}{\alpha_{k}}}\right) \mathrm{d} r_{1},
\end{aligned}
$$

where (a) follows from PPP's void probability [26] to obtain the probability density function (PDF) of $R_{k},(b)$ is due to the independence between $h_{m, 1}$ and $g_{m, 1}$, and $(c)$ follows the complementary cumulative distribution function (CCDF) of exponential distribution and the realization of $\Psi$ according to Table 1 . Also note that the approximation in $(b)$ neglects the correlation of interference set but keeps the correlation of transmission distances. We will confirm the accuracy of this approximation in Section 5. The Laplace transform $\mathrm{L}_{\Phi_{1}}(s)$ of the aggregate interference can be derived as

$$
\begin{aligned}
\mathrm{L}_{\Phi_{1}}(s) & =\mathrm{E}_{\Phi_{1}}\left[\exp \left(-s \sum_{\Phi_{1} \backslash y_{m, 1}} P_{1} h_{j, 1} Z_{j, 1}^{-\alpha_{1}}\right)\right] \\
& \stackrel{(a)}{=} \mathrm{E}_{\Phi_{1}}\left[\prod_{\Phi_{1} y_{y_{m}}} \mathrm{E}_{h_{j, 1}}\left[\exp \left(-s P_{1} h_{j, 1} Z_{j, 1}^{-\alpha_{1}}\right)\right]\right] \\
& \stackrel{(b)}{=} \exp \left(-2 \pi \lambda_{1} \int_{n}^{\infty}\left(1-\frac{1}{1+s P_{1} u^{-\alpha_{1}}}\right) u \mathrm{~d} u\right),
\end{aligned}
$$

where $(a)$ follows from the independence between $h_{j, 1}$ of each points in $\Phi_{1}$, and $(b)$ follows from the probability generating functional (PGFL) of homogeneous PPP [26]. By plugging in $s=\frac{T_{\mathrm{D}} r_{1}^{\alpha_{1}}}{P_{1}}$ and employing a change of variables $v=u^{2}\left(s P_{1}\right)^{-\frac{2}{\alpha_{1}}}$, we have 


$$
\mathrm{L}_{\Phi_{1}}\left(\frac{T_{\mathrm{D}} r_{1}^{\alpha_{1}}}{P_{1}}\right)=\exp \left(-\pi \lambda_{1} r_{1}^{2} \mathrm{G}\left(T_{\mathrm{D}}, \alpha_{1}, 1\right)\right)
$$

For $\mathrm{L}_{\Phi_{1}}\left(\frac{T_{\mathrm{C}} r_{1}^{\alpha_{1}}}{P_{1}}\right)$, similar result can be obtained simply by substituting $T_{\mathrm{D}}$ with $T_{\mathrm{C}}$. Plugging (12) into (10) and combining with (9), we reach the desired result in (8).

Since DC-UE treats data channel in the same manner as SC-UE, $\mathrm{P}_{\mathrm{C} \mid \mathrm{M}}^{(\mathrm{DC})}$ and $\mathrm{P}_{\mathrm{C} \mid \mathrm{M}}^{(\mathrm{SC})}$ will share a same definition expression, making (8) holds for both $\mathrm{P}_{\mathrm{C} \mid \mathrm{M}}^{(\mathrm{DC})}$ and $\mathrm{P}_{\mathrm{C} \mid \mathrm{M}}^{(\mathrm{SC})}$.

To obtain the unconditional data-control joint coverage probability, we need to investigate the data-control joint coverage probabilities of SC-SUEs and DC-SUEs. Denote by $\mathrm{P}_{\mathrm{CIS}}^{(\mathrm{SC})}$ and $\mathrm{P}_{\mathrm{C} \mid \mathrm{S}}^{(\mathrm{DC})}$ the conditional joint coverage probabilities of SC-SUE $\mathrm{S}_{0}$ and DC-SUE $\mathrm{E}_{0}$, respectively. We have $\quad \mathrm{P}_{\mathrm{C} \mid \mathrm{S}}^{(\mathrm{SC})}=\mathrm{P}\left(\mathrm{S}_{\mathrm{D}}^{(\mathrm{S})} \geq T_{\mathrm{D}}, \mathrm{S}_{\mathrm{C}}^{(\mathrm{S})} \geq T_{\mathrm{C}} \mid n=2\right) \quad$ and $\quad \mathrm{P}_{\mathrm{C} \mid \mathrm{S}}^{(\mathrm{DC})}=\mathrm{P}\left(\mathrm{S}_{\mathrm{D}}^{(\mathrm{S})} \geq T_{\mathrm{D}}, \mathrm{S}_{\mathrm{C}}^{(\mathrm{M})} \geq T_{\mathrm{C}} \mid n=2\right) \quad$. The expressions of $\mathrm{P}_{\mathrm{Cl}}^{(\mathrm{SC})}$ and $\mathrm{P}_{\mathrm{Cls}}^{(\mathrm{DC})}$ are provided in Lemma 2.

Lemma 2. In HCN, the conditional joint coverage probabilities of a typical SC-UE and a DC-UE receiving data signals from SeNBs under multi-frequency tiering are given by

$$
\begin{aligned}
\mathrm{P}_{\mathrm{C} \mid \mathrm{S}}^{(\mathrm{SC})} & =\frac{2 \pi \lambda_{2}}{\mathrm{~A}_{2}} \int_{0}^{\infty} r \exp \left(-\pi \lambda_{2} r^{2}\left(\mathrm{G}\left(T_{\mathrm{C}}, \alpha_{2}, 1\right)+\mathrm{G}\left(T_{\mathrm{D}}, \alpha_{2}, 1\right)\right)\right) \\
& \times \exp \left(-\frac{r^{\alpha_{2}} \sigma^{2}\left(T_{\mathrm{D}}+T_{\mathrm{C}}\right)}{P_{2}}-\pi \sum_{k=1}^{2} \lambda_{k}\left(\frac{P_{k} B_{k}}{P_{2} B_{2}}\right)^{\frac{2}{\alpha_{k}}} r^{\frac{2 \alpha_{2}}{\alpha_{k}}}\right) \mathrm{d} r,
\end{aligned}
$$

and

$$
\begin{aligned}
\mathrm{P}_{\mathrm{C} \mathrm{S} S}^{(\mathrm{DC})}= & \frac{4 \pi^{2} \lambda_{1} \lambda_{2}}{\mathrm{~A}_{2}^{2}} \int_{0}^{\infty} \exp \left(-\frac{r_{2}^{\alpha_{2}} \sigma^{2} T_{\mathrm{D}}}{P_{2}}-\pi \lambda_{2} r_{2}^{2} \mathrm{G}\left(T_{\mathrm{D}}, \alpha_{2}, 1\right)-\pi \sum_{k=1}^{2} \lambda_{k}\left(\frac{P_{k} B_{k}}{P_{2} B_{2}}\right)^{\frac{2}{\alpha_{k}}} r_{2}^{\frac{2 \alpha_{2}}{\alpha_{k}}}\right) r_{2} \mathrm{~d} r_{2} \\
& \times \int_{0}^{\infty} \exp \left(-\frac{r_{1}^{\alpha_{1}} \sigma^{2} T_{\mathrm{C}}}{P_{1}}-\pi \lambda_{1} r_{1}^{2}\left(\mathrm{G}\left(T_{\mathrm{C}}, \alpha_{1}, 1\right)+1\right)\right) \cdot\left(1-\exp \left(-\pi \lambda_{2}\left(\frac{P_{2} B_{2}}{P_{1} B_{1}}\right)^{\frac{2}{\alpha_{2}}} r_{1}^{\frac{2 \alpha_{1}}{\alpha_{2}}}\right)\right) r_{1} \mathrm{~d} r_{1},
\end{aligned}
$$

where $A_{2}$ is given in (7).

Proof: Comparing Fig. 2(a) and Fig. 2(b), one can observe that SC-SUE $E_{0}$ treats data and control channel in a way that is similar to $\mathrm{SC}-\mathrm{MUE}_{0}$ by connecting both channels to the same eNB. The only difference lies in the type of eNB providing the desired signal. Accordingly, the expression of $\mathrm{P}_{\mathrm{CSS}}^{(\mathrm{SC})}$ in (13) can be reached by applying the same derivation in Lemma 1.

As for the conditional joint coverage probability of DC-UE $E_{0}$ receiving data signals from SeNBs, $\mathrm{P}_{\mathrm{C} \mid \mathrm{S}}^{(\mathrm{DC})}$ is given by

$$
\begin{aligned}
\mathrm{P}_{\mathrm{ClS}}^{(\mathrm{DC})} & =\mathrm{P}\left(\mathrm{S}_{\mathrm{D}}^{(\mathrm{S})} \geq T_{\mathrm{D}}, \mathrm{S}_{\mathrm{C}}^{(\mathrm{M})} \geq T_{\mathrm{C}} \mid n=2\right) \\
& \approx \mathrm{P}\left(\mathrm{S}_{\mathrm{D}}^{(\mathrm{S})} \geq T_{\mathrm{D}} \mid n=2\right) \mathrm{P}\left(\mathrm{S}_{\mathrm{C}}^{(\mathrm{M})} \geq T_{\mathrm{C}} \mid n=2\right) \\
& =\frac{\mathrm{P}\left(\mathrm{S}_{\mathrm{D}}^{(\mathrm{S})} \geq T_{\mathrm{D}}, n=2\right)}{\mathrm{P}(n=2)} \cdot \frac{\mathrm{P}\left(\mathrm{S}_{\mathrm{C}}^{(\mathrm{M})} \geq T_{\mathrm{C}}, n=2\right)}{\mathrm{P}(n=2)},
\end{aligned}
$$

where $\mathrm{P}(n=2)=\mathrm{A}_{2}$. The approximation in $(a)$ neglects part of the correlation between the transmission distances of data channel and control channel (i.e., $R_{1}$ and $R_{2}$ ). However, unlike 
the strong correlation of same transmission distances for SC-MUE ${ }_{0}, \mathrm{SC}-\mathrm{SUE}_{0}$ and DC-MUE this correlation is greatly weakened for DC-SUE $E_{0}$ with different $R_{1}$ and $R_{2}$. We will confirm the accuracy of this approximation in Section 5. In (15), $\mathrm{P}\left(\mathrm{S}_{\mathrm{D}}^{(\mathrm{s})} \geq T_{\mathrm{D}}, n=2\right)$ can be derived as

$$
\begin{aligned}
\mathrm{P}\left(\mathrm{S}_{\mathrm{D}}^{(\mathrm{S})} \geq T_{\mathrm{D}}, n=2\right) & =\int_{0}^{\infty} \mathrm{P}\left(\mathrm{S}_{\mathrm{D}}^{(\mathrm{S})} \geq T_{\mathrm{D}}, R_{1}>\left(\frac{P_{1} B_{1}}{P_{2} B_{2}}\right)^{\frac{1}{\alpha_{1}}} r_{2}^{\frac{\alpha_{2}}{\alpha_{1}}}\right) \cdot 2 \pi \lambda_{2} r_{2} \exp \left(-\lambda_{2} \pi r_{2}^{2}\right) \mathrm{d} r_{2} \\
& =2 \pi \lambda_{2} \int_{0}^{\infty} \mathrm{P}\left(\mathrm{S}_{\mathrm{D}}^{(\mathrm{S})} \geq T_{\mathrm{D}}\right) \exp \left(-\lambda_{1} \pi\left(\frac{P_{1} B_{1}}{P_{2} B_{2}}\right)^{\frac{2}{\alpha_{1}}} r_{2}^{\frac{2 \alpha_{2}}{\alpha_{1}}}\right) \cdot r_{2} \exp \left(-\lambda_{2} \pi r_{2}^{2}\right) \mathrm{d} r_{2},
\end{aligned}
$$

where $\mathrm{P}\left(\mathrm{S}_{\mathrm{D}}^{(\mathrm{s})} \geq T_{\mathrm{D}}\right)=\mathrm{P}\left(h_{m, 2} \geq \frac{T_{\mathrm{D}} r_{2}^{\alpha_{2}}}{P_{2}}\left(\sum_{\Psi \backslash y_{m, 2}} P_{k} h_{j, k} Z_{j, k}^{-\alpha_{k}}+\sigma^{2}\right)\right)$ can be evaluated in the same way as Lemma 1 and is given by

$$
\mathrm{P}\left(\mathrm{S}_{\mathrm{D}}^{(\mathrm{s})} \geq T_{\mathrm{D}}\right)=\exp \left(-\frac{T_{\mathrm{D}} r_{2}^{\alpha_{2}} \sigma^{2}}{P_{2}}-\pi \lambda_{2} r_{2}^{2} \mathrm{G}\left(T_{\mathrm{D}}, \alpha_{2}, 1\right)\right) .
$$

Meanwhile, $\mathrm{P}\left(\mathrm{S}_{\mathrm{C}}^{(\mathrm{M})} \geq T_{\mathrm{C}}, n=2\right)$ can be derived as

$$
\begin{aligned}
& \mathrm{P}\left(\mathrm{S}_{\mathrm{C}}^{(\mathrm{M})} \geq T_{\mathrm{C}}, n=2\right) \\
= & \int_{0}^{\infty} \mathrm{P}\left(\mathrm{S}_{\mathrm{C}}^{(\mathrm{M})} \geq T_{\mathrm{C}}, R_{2}<\left(\frac{P_{2} B_{2}}{P_{1} B_{1}}\right)^{\frac{1}{\alpha_{2}}} r_{1}^{\frac{\alpha_{1}}{\alpha_{2}}}\right) \cdot 2 \pi \lambda_{1} r_{1} \exp \left(-\lambda_{1} \pi r_{1}^{2}\right) \mathrm{d} r_{1} \\
= & 2 \pi \lambda_{1} \int_{0}^{\infty} \mathrm{P}\left(\mathrm{S}_{\mathrm{C}}^{(\mathrm{M})} \geq T_{\mathrm{C}}\right)\left(1-\exp \left(-\pi \lambda_{2}\left(\frac{P_{2} B_{2}}{P_{1} B_{1}}\right)^{\frac{2}{\alpha_{2}}} r_{1}^{\frac{2 \alpha_{1}}{\alpha_{2}}}\right)\right) \cdot r_{1} \exp \left(-\lambda_{1} \pi r_{1}^{2}\right) \mathrm{d} r_{1},
\end{aligned}
$$

where $\mathrm{P}\left(\mathrm{S}_{\mathrm{C}}^{(\mathrm{M})} \geq T_{\mathrm{C}}\right)=\mathrm{P}\left(g_{m, 1} \geq \frac{T_{\mathrm{C}} r_{1}^{\alpha_{1}}}{P_{1}}\left(\sum_{\Psi y_{m, 1}} P_{k} h_{j, k} Z_{j, k}^{-\alpha_{k}}+\sigma^{2}\right)\right)$ can also be evaluated in the same way as Lemma 1 and is given by

$$
\mathrm{P}\left(\mathrm{S}_{\mathrm{C}}^{(\mathrm{M})} \geq T_{\mathrm{C}}\right)=\exp \left(-\frac{T_{\mathrm{C}} r_{1}^{\alpha_{1}} \sigma^{2}}{P_{1}}-\pi \lambda_{1} r_{1}^{2} \mathrm{G}\left(T_{\mathrm{C}}, \alpha_{1}, 1\right)\right) .
$$

Plugging (17) and (19) into (16) and (18), and combining with (15) along with some algebraic manipulations, we reach the desired result in (14).

Although Lemma 1 and Lemma 2 does not give closed-form expressions, the single integral is not difficult to compute. Note that (14) is calculated by multiplying two single integrals instead of one double integral. We will also show in Corollary 1 that, in some special cases, the expressions in Lemma 1 and Lemma 2 will admit simple closed-forms.

\subsection{Overall Joint Coverage Probabilities under Multi-Frequency Tiering}

Finally, the overall joint coverage probability can be achieved from the law of total probability and is given in the following Theorem 1 .

Theorem 1. In HCN, the overall data-control joint coverage probabilities of a typical SC-UE and a DC-UE under multi-frequency tiering are given by 


$$
\begin{aligned}
\mathrm{P}_{\mathrm{C}}^{(\mathrm{sc})}= & 2 \pi \sum_{n=1}^{2} \lambda_{n} \int_{0}^{\infty} r \exp \left(-\pi \lambda_{n} r^{2}\left(\mathrm{G}\left(T_{\mathrm{C}}, \alpha_{n}, 1\right)+\mathrm{G}\left(T_{\mathrm{D}}, \alpha_{n}, 1\right)\right)\right) \\
& \times \exp \left(-\frac{r^{\alpha_{n}} \sigma^{2}\left(T_{\mathrm{D}}+T_{\mathrm{C}}\right)}{P_{n}}-\pi \sum_{k=1}^{2} \lambda_{k}\left(\frac{P_{k} B_{k}}{P_{n} B_{n}}\right)^{\frac{2}{\alpha_{k}}} r^{\frac{2 \alpha_{n}}{\alpha_{k}}}\right) \mathrm{d} r,
\end{aligned}
$$

and

$$
\begin{aligned}
\mathrm{P}_{\mathrm{C}}^{(\mathrm{DC})}= & 2 \pi \lambda_{1} \int_{0}^{\infty} r \exp \left(-\pi \lambda_{1} r^{2}\left(\mathrm{G}\left(T_{\mathrm{C}}, \alpha_{1}, 1\right)+\mathrm{G}\left(T_{\mathrm{D}}, \alpha_{1}, 1\right)\right)\right) \\
& \times \exp \left(-\frac{r^{\alpha_{1}} \sigma^{2}\left(T_{\mathrm{D}}+T_{\mathrm{C}}\right)}{P_{1}}-\pi \sum_{k=1}^{2} \lambda_{k}\left(\frac{P_{k} B_{k}}{P_{1} B_{1}}\right)^{\frac{2}{\alpha_{k}}} r^{\frac{2 \alpha_{1}}{\alpha_{k}}}\right) \mathrm{d} r \\
& +\frac{4 \pi^{2} \lambda_{1} \lambda_{2}}{\mathrm{~A}_{2}} \int_{0}^{\infty} \exp \left(-\frac{r_{2}^{\alpha_{2}} \sigma^{2} T_{\mathrm{D}}}{P_{2}}-\pi \lambda_{2} r_{2}^{2} \mathrm{G}\left(T_{\mathrm{D}}, \alpha_{2}, 1\right)-\pi \sum_{k=1}^{2} \lambda_{k}\left(\frac{P_{k} B_{k}}{P_{2} B_{2}}\right)^{\frac{2}{\alpha_{k}}} r_{2}^{\frac{2 \alpha_{2}}{\alpha_{k}}}\right) r_{2} \mathrm{~d} r_{2} \\
& \times \int_{0}^{\infty} \exp \left(-\frac{r_{1}^{\alpha_{1}} \sigma^{2} T_{\mathrm{C}}}{P_{1}}-\pi \lambda_{1} r_{1}^{2}\left(\mathrm{G}\left(T_{\mathrm{C}}, \alpha_{1}, 1\right)+1\right)\right) \cdot\left(1-\exp \left(-\pi \lambda_{2}\left(\frac{P_{2} B_{2}}{P_{1} B_{1}}\right)^{\frac{2}{\alpha_{2}}} r_{1}^{\frac{2 \alpha_{1}}{\alpha_{2}}}\right)\right) r_{1} \mathrm{~d} r_{1} .
\end{aligned}
$$

In HCN, the interference power is far higher than the thermal noise power due to dense eNB deployment, so we neglect thermal noise and consider a special case of equal path loss exponents in Corollary 1, which provides closed-form expressions for the joint coverage probabilities.

Corollary 1. When $\alpha_{k}=\alpha$ and thermal noise is neglected (i.e., $\sigma^{2}=0$ ), the joint coverage probabilities of a DC-UE and a SC-UE under multi-frequency tiering are given by

$$
\mathrm{P}_{\mathrm{C}}^{(\mathrm{SC})}=\frac{1}{\mathrm{G}\left(T_{\mathrm{C}}, \alpha, 1\right)+\mathrm{G}\left(T_{\mathrm{D}}, \alpha, 1\right)+1+u}+\frac{u}{u\left(\mathrm{G}\left(T_{\mathrm{C}}, \alpha, 1\right)+\mathrm{G}\left(T_{\mathrm{D}}, \alpha, 1\right)+1\right)+1},
$$

and

$$
\begin{aligned}
P_{C}^{(\mathrm{DC})}= & \frac{1}{\mathrm{G}\left(T_{\mathrm{C}}, \alpha, 1\right)+\mathrm{G}\left(T_{\mathrm{D}}, \alpha, 1\right)+1+u} \\
& +\frac{1}{\mathrm{G}\left(T_{\mathrm{C}}, \alpha, 1\right)+1} \cdot \frac{u}{\mathrm{G}\left(T_{\mathrm{C}}, \alpha, 1\right)+1+u} \cdot \frac{1+u}{1+u\left(\mathrm{G}\left(T_{\mathrm{D}}, \alpha, 1\right)+1\right)},
\end{aligned}
$$

where $u=\frac{\lambda_{2}}{\lambda_{1}}\left(\frac{P_{2} B_{2}}{P_{1} B_{1}}\right)^{\frac{2}{\alpha}}$.

From Corollary 1, we can observed that the overall maximum joint coverage probability is decided by $T_{\mathrm{D}}, T_{\mathrm{C}}$ and the path loss exponent $\alpha$. Once these parameters are set, the densities of eNBs, transmit powers and cell association biases will not influence the achievable global maximum value, which will be illustrated and further discussed in Section 5.

\section{SINR Performance Analysis under Single-Frequency Tiering}

In this section, we extend our SINR characterization to the single-frequency tiering case where MeNBs and SeNBs transmit on the same spectrum. As concluded in Table 1, the interference sets for SC-MUE, SC-SUE, DC-MUE, and DC-SUE under single-frequency tiering all comprise interference sources from both tiers. Therefore, both data and control channels will 
suffer more sophisticated and severe interference. Similar methodology in Section 3 can be followed by investigating the conditional joint coverage probabilities.

\subsection{Conditional Joint Coverage Probabilities under Single-Frequency Tiering}

To better understand how Lemma 1 and Lemma 2 are extended to the single-frequency tiering case, we will firstly recall the difference between single-frequency tiering and multi-frequency tiering from the aspects of interference set and transmission distance.

Under multi-frequency tiering, with $y_{m, n}$ being the nearest eNB within the accessed spectrum, the power of the received signal from $y_{m, n}$ is higher than any other interfering eNB, guaranteeing a relatively high SINR for both data and control channel. However, under single-frequency tiering, SINR may deteriorate not only because of the increased amount of interfering eNBs, but also due to the biased association criteria described in (1) allowing $y_{m, n}$ not being the nearest one within the accessed spectrum. What is worse for DC-SUEs, the interference on control channel can be extremely severe. This is because in this case $y_{m, 2}$ is playing the role of interfering eNB to the serving eNB $y_{m, 1}$ for the control channel and being a SUE implies a relatively small $R_{2}$ making $y_{m, 2}$ even closer than $y_{m, 1}$.

Similar to Lemma 1 and Lemma 2, we derive the expressions of $\mathrm{P}_{\mathrm{C} \mid \mathrm{M}}^{(\mathrm{SC})}$ and $\mathrm{P}_{\mathrm{C} \mid \mathrm{M}}^{(\mathrm{DC})}$ in Lemma 3 and the expressions of $\mathrm{P}_{\mathrm{C} \mid \mathrm{s}}^{(\mathrm{SC})}$ and $\mathrm{P}_{\mathrm{C} \mid \mathrm{S}}^{(\mathrm{DC})}$ in Lemma 4 under single-frequency tiering.

Lemma 3. In HCN, the conditional joint coverage probabilities of a typical SC-UE and a DC-UE receiving data signals from MeNBs under single-frequency tiering are given by

$$
\begin{aligned}
\mathrm{P}_{\mathrm{C} \mid \mathrm{M}}^{(\mathrm{DC})}=\mathrm{P}_{\mathrm{C} \mid \mathrm{M}}^{(\mathrm{SC})} & =\frac{2 \pi \lambda_{1}}{\mathrm{~A}_{1}} \int_{0}^{\infty} r \exp \left(-\frac{r^{\alpha_{1}} \sigma^{2}\left(T_{\mathrm{D}}+T_{\mathrm{C}}\right)}{P_{1}}\right) \\
& \times \exp \left(-\pi \sum_{k=1}^{2} \lambda_{k}\left(\frac{P_{k}}{P_{1}}\right)^{\frac{2}{\alpha_{k}}} r^{\frac{2 \alpha_{1}}{\alpha_{k}}}\left(\mathrm{G}\left(T_{\mathrm{C}}, \alpha_{k}, \frac{B_{k}}{B_{1}}\right)+\mathrm{G}\left(T_{\mathrm{D}}, \alpha_{k}, \frac{B_{k}}{B_{1}}\right)+\left(\frac{B_{k}}{B_{1}}\right)^{\frac{2}{\alpha_{k}}}\right)\right) \mathrm{d} r .
\end{aligned}
$$

Proof: As concluded in Table 1, the difference between multi-frequency tiering and single-frequency tiering for a MUE mainly lies in the constitution of the interference set. Therefore, by substituting $\Phi_{1}+\Phi_{2}$ into $\Psi, \mathrm{P}_{\mathrm{C} \mid \mathrm{M}}^{(\mathrm{SC})}$ and $\mathrm{P}_{\mathrm{C} \mid \mathrm{M}}^{(\mathrm{DC})}$ can be obtained by applying similar derivations as (10), which is given by

$$
\begin{aligned}
\mathrm{P}_{\mathrm{C} \mid \mathrm{M}}^{(\mathrm{SC})} & =\mathrm{P}_{\mathrm{C} \mid \mathrm{M}}^{(\mathrm{DC})}=\mathrm{P}\left(\mathrm{S}_{\mathrm{D}}^{(\mathrm{M})} \geq T_{\mathrm{D}}, \mathrm{S}_{\mathrm{C}}^{(\mathrm{M})} \geq T_{\mathrm{C}}, n=1\right) \\
& =2 \pi \lambda_{1} \int_{0}^{\infty} \mathrm{L}_{\Phi_{1}}\left(\frac{T_{\mathrm{D}} r_{1}^{\alpha_{1}}}{P_{1}}\right) \mathrm{L}_{\Phi_{2}}\left(\frac{T_{\mathrm{D}} r_{1}^{\alpha_{1}}}{P_{1}}\right) \mathrm{L}_{\Phi_{1}}\left(\frac{T_{\mathrm{C}} r_{1}^{\alpha_{1}}}{P_{1}}\right) \mathrm{L}_{\Phi_{2}}\left(\frac{T_{\mathrm{C}} r_{1}^{\alpha_{1}}}{P_{1}}\right) \\
& \times r_{1} \exp \left(-\frac{r_{1}^{\alpha_{1}} \sigma^{2}\left(T_{\mathrm{D}}+T_{\mathrm{C}}\right)}{P_{1}}-\pi \sum_{k=1}^{2} \lambda_{k}\left(\frac{P_{k} B_{k}}{P_{1} B_{1}}\right)^{\frac{2}{\alpha_{k}}} r_{1}^{\frac{2 \alpha_{1}}{\alpha_{k}}}\right) \mathrm{d} r_{1},
\end{aligned}
$$

where the Laplace transform $\mathrm{L}_{\Phi_{2}}\left(\frac{T_{\mathrm{D}} r_{1}^{\alpha_{1}}}{P_{1}}\right)$ can be evaluated through (12) and is given by

$$
\mathrm{L}_{\Phi_{2}}\left(\frac{T_{\mathrm{D}} r_{1}^{\alpha_{1}}}{P_{1}}\right)=\exp \left(-\pi \lambda_{2} r_{1}^{\frac{2 \alpha_{1}}{\alpha_{2}}}\left(\frac{P_{2}}{P_{1}}\right)^{\frac{2}{\alpha_{2}}} \mathrm{G}\left(T_{\mathrm{D}}, \alpha_{2}, \frac{B_{2}}{B_{1}}\right)\right) \text {. }
$$


For $\mathrm{L}_{\Phi_{2}}\left(\frac{T_{\mathrm{C}} r_{1}^{\alpha_{1}}}{P_{1}}\right)$, similar result can be obtained simply by substituting $T_{\mathrm{D}}$ with $T_{\mathrm{C}}$.

Lemma 4. In HCN, the conditional joint coverage probabilities of a typical SC-UE and a DC-UE receiving data signals from SeNBs under single-frequency tiering are given by

$$
\begin{aligned}
\mathrm{P}_{\mathrm{CIS}}^{(\mathrm{SC})} & =\frac{2 \pi \lambda_{2}}{\mathrm{~A}_{2}} \int_{0}^{\infty} r \exp \left(-\frac{r^{\alpha_{2}} \sigma^{2}\left(T_{\mathrm{D}}+T_{\mathrm{C}}\right)}{P_{2}}\right) \\
& \times \exp \left(-\pi \sum_{k=1}^{2} \lambda_{k}\left(\frac{P_{k}}{P_{2}}\right)^{\frac{2}{\alpha_{k}}} r^{\frac{2 \alpha_{2}}{\alpha_{k}}}\left(\mathrm{G}\left(T_{\mathrm{C}}, \alpha_{k}, \frac{B_{k}}{B_{2}}\right)+\mathrm{G}\left(T_{\mathrm{D}}, \alpha_{k}, \frac{B_{k}}{B_{2}}\right)+\left(\frac{B_{k}}{B_{2}}\right)^{\frac{2}{\alpha_{k}}}\right)\right) \mathrm{d} r,
\end{aligned}
$$

and

$$
\begin{aligned}
\mathrm{P}_{\mathrm{C} \mid \mathrm{S}}^{(\mathrm{DC})} & =\frac{4 \pi^{2} \lambda_{1} \lambda_{2}}{\mathrm{~A}_{2}^{2}} \int_{0}^{\infty} \exp \left(-\frac{r_{2}^{\alpha_{2}} \sigma^{2} T_{\mathrm{D}}}{P_{2}}-\pi \sum_{k=1}^{2} \lambda_{k}\left(\frac{P_{k}}{P_{2}}\right)^{\frac{2}{\alpha_{k}}} r_{2}^{\frac{2 \alpha_{2}}{\alpha_{k}}}\left(\left(\frac{B_{k}}{B_{2}}\right)^{\frac{2}{\alpha_{k}}}+\mathrm{G}\left(T_{\mathrm{D}}, \alpha_{k}, \frac{B_{k}}{B_{2}}\right)\right) r_{2} \mathrm{~d} r_{2}\right. \\
& \times \int_{0}^{\infty} r_{1} \exp \left(-\frac{r_{1}^{\alpha_{1}} \sigma^{2} T_{\mathrm{C}}}{P_{1}}\right)\left[\exp \left(-\pi \lambda_{2} r_{1}^{\frac{2 \alpha_{1}}{\alpha_{2}}}\left(\frac{P_{2}}{P_{1}}\right)^{\frac{2}{\alpha_{2}}} T_{\mathrm{C}}^{\frac{2}{\alpha_{2}}} \frac{2 \pi / \alpha_{2}}{\sin \left(2 \pi / \alpha_{2}\right)}\right)\right. \\
& \left.-\exp \left(-\pi \lambda_{2}\left(\frac{P_{2}}{P_{1}}\right)^{\frac{2}{\alpha_{2}}} r_{1}^{\frac{2 \alpha_{1}}{\alpha_{2}}}\left(\mathrm{G}\left(T_{\mathrm{C}}, \alpha_{2}, \frac{B_{2}}{B_{1}}\right)+\left(\frac{B_{2}}{B_{1}}\right)^{\frac{2}{\alpha_{2}}}\right)\right)\right] \exp \left(-\pi \lambda_{1} r_{1}^{2}\left(\mathrm{G}\left(T_{\mathrm{C}}, \alpha_{1}, 1\right)+1\right)\right) \mathrm{d} r_{1} .
\end{aligned}
$$

Proof: Following similar extensions from Lemma 1 to Lemma 2 and Lemma 3, the expression of $\mathrm{P}_{\mathrm{Cl} S \mathrm{~S}}^{\mathrm{sC})}$ in (27) can be easily obtained.

As for $\mathrm{P}_{\mathrm{C} \mid \mathrm{s}}^{(\mathrm{DC})}$ under multi-frequency tiering, applying similar dirivations as (15) - (19), the expression in (28) can be achieved. Note that the Laplace transform for $\Phi_{2}$ on control channel is evaluated as

$$
\mathrm{L}_{\Phi_{2}}\left(\frac{T_{\mathrm{D}} r_{1}^{\alpha_{1}}}{P_{1}}\right)=\exp \left(-\pi \lambda_{2} r_{1}^{\frac{2 \alpha_{1}}{\alpha_{2}}}\left(\frac{P_{2}}{P_{1}}\right)^{\frac{2}{\alpha_{2}}} T_{\mathrm{C}}^{\frac{2}{\alpha_{2}}} \frac{2 \pi / \alpha_{2}}{\sin \left(2 \pi / \alpha_{2}\right)}\right),
$$

which is due to the fact that the interference set for DC-SUE $E_{0}$ not only expands from $\Phi_{1}$ to $\Phi_{1}+\Phi_{2}$, but also includes interfering SeNB that may be any close to the DC-SUE .

Similar to Lemma 1 and Lemma 2, the single integrals in Lemma 3 and Lemma 4 are not difficult to compute.

\subsection{Overall Joint Coverage Probabilities under Single-Frequency Tiering}

Following the law of total probability, Theorem 2 provides the overall joint coverage probabilities for UE with and without DC capability.

Theorem 2. In HCN, the overall data-control joint coverage probabilities of a typical SC-UE and a DC-UE under single-frequency tiering are given by 


$$
\begin{aligned}
\mathrm{P}_{\mathrm{C}}^{(\mathrm{SC})}= & 2 \pi \sum_{n=1}^{2} \lambda_{n} \int_{0}^{\infty} r \exp \left(-\frac{r^{\alpha_{n}} \sigma^{2}\left(T_{\mathrm{D}}+T_{\mathrm{C}}\right)}{P_{n}}\right) \\
& \times \exp \left(-\pi \sum_{k=1}^{2} \lambda_{k}\left(\frac{P_{k}}{P_{n}}\right)^{\frac{2}{\alpha_{k}}} r^{\frac{2 \alpha_{n}}{\alpha_{k}}}\left(\mathrm{G}\left(T_{\mathrm{C}}, \alpha_{k}, \frac{B_{k}}{B_{n}}\right)+\mathrm{G}\left(T_{\mathrm{D}}, \alpha_{k}, \frac{B_{k}}{B_{n}}\right)+\left(\frac{B_{k}}{B_{n}}\right)^{\frac{2}{\alpha_{k}}}\right)\right) \mathrm{d} r,
\end{aligned}
$$

and

$$
\begin{aligned}
\mathrm{P}_{\mathrm{C}}^{(\mathrm{DC})} & =2 \pi \lambda_{1}^{\infty} \int_{0}^{\infty} r \exp \left(-\frac{r^{\alpha_{1}} \sigma^{2}\left(T_{\mathrm{D}}+T_{\mathrm{C}}\right)}{P_{1}}\right) \\
& \times \exp \left(-\pi \sum_{k=1}^{2} \lambda_{k}\left(\frac{P_{k}}{P_{1}}\right)^{\frac{2}{\alpha_{k}}} r^{\frac{2 \alpha_{1}}{\alpha_{k}}}\left(\mathrm{G}\left(T_{\mathrm{C}}, \alpha_{k}, \frac{B_{k}}{B_{1}}\right)+\mathrm{G}\left(T_{\mathrm{D}}, \alpha_{k}, \frac{B_{k}}{B_{1}}\right)+\left(\frac{B_{k}}{B_{1}}\right)^{\frac{2}{\alpha_{k}}}\right)\right) \mathrm{d} r \\
& +\frac{4 \pi^{2} \lambda_{1} \lambda_{2}}{\mathrm{~A}_{2}} \int_{0}^{\infty} \exp \left(-\frac{r_{2}^{\alpha_{2}} \sigma^{2} T_{\mathrm{D}}}{P_{2}}-\pi \sum_{k=1}^{2} \lambda_{k}\left(\frac{P_{k}}{P_{2}}\right)^{\frac{2}{\alpha_{k}}} r_{2}^{\frac{2 \alpha_{2}}{\alpha_{k}}}\left(\left(\frac{B_{k}}{B_{2}}\right)^{\frac{2}{\alpha_{k}}}+\mathrm{G}\left(T_{\mathrm{D}}, \alpha_{k}, \frac{B_{k}}{B_{2}}\right)\right)\right) r_{2} \mathrm{~d} r_{2} \\
& \times \int_{0}^{\infty} r_{1} \exp \left(-\frac{r_{1}^{\alpha_{1}} \sigma^{2} T_{\mathrm{C}}}{P_{1}}\right)\left[\exp \left(-\pi \lambda_{2} r_{1}^{\frac{2 \alpha_{1}}{\alpha_{2}}}\left(\frac{P_{2}}{P_{1}}\right)^{\frac{2}{\alpha_{2}}} \frac{2}{T_{\mathrm{C}}^{\frac{2}{\alpha_{2}}}} \frac{2 \pi / \alpha_{2}}{\sin \left(2 \pi / \alpha_{2}\right)}\right)\right. \\
& \left.-\exp \left(-\pi \lambda_{2}\left(\frac{P_{2}}{P_{1}}\right)^{\frac{2}{\alpha_{2}}} r_{1}^{\frac{2 \alpha_{1}}{\alpha_{2}}}\left(\mathrm{G}\left(T_{\mathrm{C}}, \alpha_{2}, \frac{B_{2}}{B_{1}}\right)+\left(\frac{B_{2}}{B_{1}}\right)^{\frac{2}{\alpha_{2}}}\right)\right)\right] \exp \left(-\pi \lambda_{1} r_{1}^{2}\left(\mathrm{G}\left(T_{\mathrm{C}}, \alpha_{1}, 1\right)+1\right)\right) \mathrm{d} r_{1} .
\end{aligned}
$$

Theorem 1 and Theorem 2 provide the main technical results of this paper. We will apply these results to investigate the DC mechanism from various aspects in the following section.

\section{Numerical Results and Discussion}

In this section, we evaluate the joint coverage performance under the proposed model. We firstly validate our analytical expressions against Monte Carlo simulations. Next, based on the developed expressions, we compare the SINR performance of DC and traditional SC. Then, we further evaluate DC mechanism under multi-frequency and single-frequency tiering scenarios. Finally, we analyze the impacts of path loss exponents and association bias over optimal eNB density ratio and the corresponding maximal joint coverage probability to unveil important insights on the network design.

We consider a 2-tier downlink HCN including MeNBs and SeNBs as described in Section 2. Unless otherwise stated, the main simulation parameters used in this section are summarized in Table 3. It should also be noted that the results based on Poisson point process provide worst-case representations since the eNB sites are carefully determined in actual deployment for better coverage and lower interference [21].

Table 3. Parameter Assumptions

\begin{tabular}{|c|c|c|}
\hline Parameter & Description & Value \\
\hline \hline$\sigma^{2}$ & Thermal noise power & $-104 \mathrm{dBm}$ \\
\hline$P_{1}$ & Transmit power for data and control channel of MeNBs & $43 \mathrm{dBm}$ \\
\hline$P_{2}$ & Transmit power for data and control channel of SeNBs & $23 \mathrm{dBm}$ \\
\hline$\alpha_{1}$ & Path loss exponent for MeNBs transmission link & 3.8 \\
\hline
\end{tabular}




\begin{tabular}{|c|c|c|}
\hline$B_{1}$ & Cell association bias for MeNBs link & 1 \\
\hline$T_{\mathrm{C}}$ & Control channel SINR threshold & $-1.6 \mathrm{~dB}$ \\
\hline$\lambda_{1}$ & Density of MeNBs & $1 \mathrm{eNB} / \mathrm{km}^{2}$ \\
\hline & Realization number of Monte Carlo simulation & $100000 \mathrm{drops}$ \\
\hline & Monte Carlo simulation area & $50 \times 50 \mathrm{~km}^{2}$ \\
\hline
\end{tabular}

\subsection{Validation of Analytical Expressions}

We firstly validate our analytical expressions in Fig. 3 and Fig. 4 by comparing the derived expressions with Monte Carlo simulation results. Note that $T_{\mathrm{C}}$ is fixed at $-1.6 \mathrm{~dB}$ in Fig. 3 and Fig. 4(a), and is variable in Fig. 4(b) to verify the control channel SINR characterization.

In Fig. 3(a) and Fig. 3(b), overall joint coverage probabilities given in (20), (21), (30), and (31) are validated with the increase of $T_{\mathrm{D}}$ and $\lambda_{2} / \lambda_{1}$. For DC-UE and SC-UE in different tiering scenarios, analytical curves closely match the simulation curves in both subfigures, which confirms the accuracy of our analytical expressions. The validity of further performance analyses in the following subsections are hereby confirmed. However, both subfigures present a more obvious gap between the curves for DC-UE under multi-frequency tiering than that of other cases. We will dig into this gap with the help of Fig. 4.

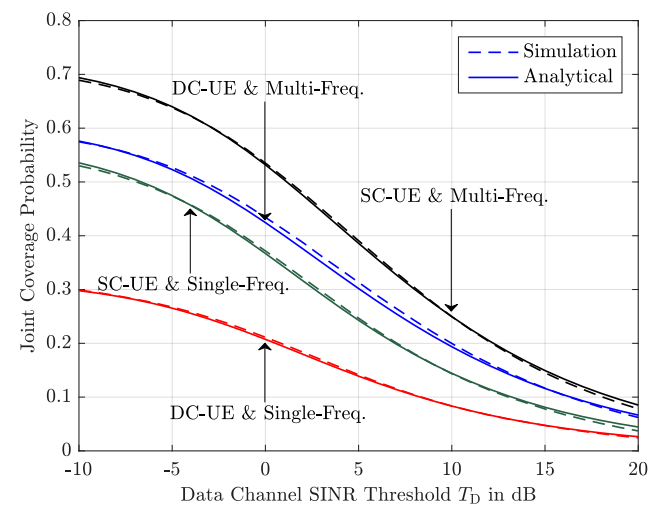

(a)

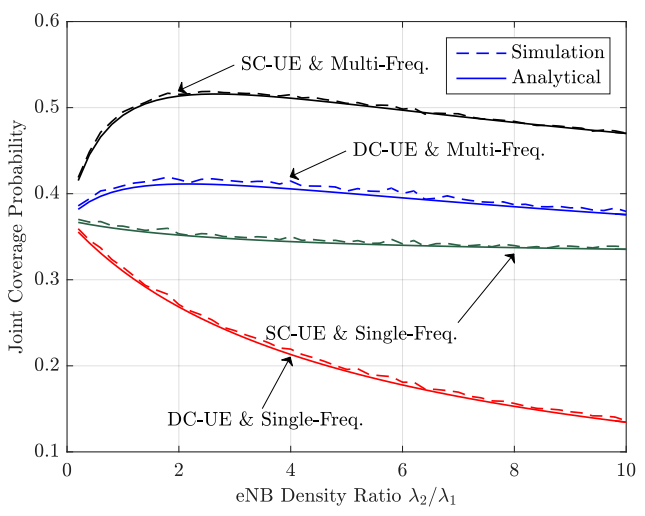

(b)

Fig. 3. Expression validation with Monte Carlo simulation when $\alpha_{2}=3.5$ and $B_{2}=2$.

In Fig. 4, deep-going observations are carried out to study the reason of the small gap in DC-UE expression under multi-frequency tiering. Fig. 4(a) decomposes overall joint coverage probabilities into conditional probabilities for MUE and SUE as given in (8), (13) and (14). The subfigure presents that the curve of (14) for DC-SUE is not that close as (8) and (13) for SC-MUE, DC-MUE and SC-SUE, which contributes to the above mentioned gap in Fig. 3 and leads us to further investigation on the derivation of (14). With (15), the conditional joint coverage probability of DC-SUE is approximated in part by the product of (16) and (18), and these two equations are both shown to match well with simulation curves in Fig. 4(b). However, when correlation is partly neglected to obtain (14) through multiplying (16) by (18), a slight extent of accuracy is dropped, bringing in the gap between the red curves in Fig. 4(b). Therefore, we can conclude that the slight accuracy loss in (14) is due to neglecting part of the correlation between transmission distances of data and control channels of DC-SUE. However, it should be noted that even with a small gap, (14) is still with sufficient accuracy and the approximation of neglecting part of the correlation is reasonable. 


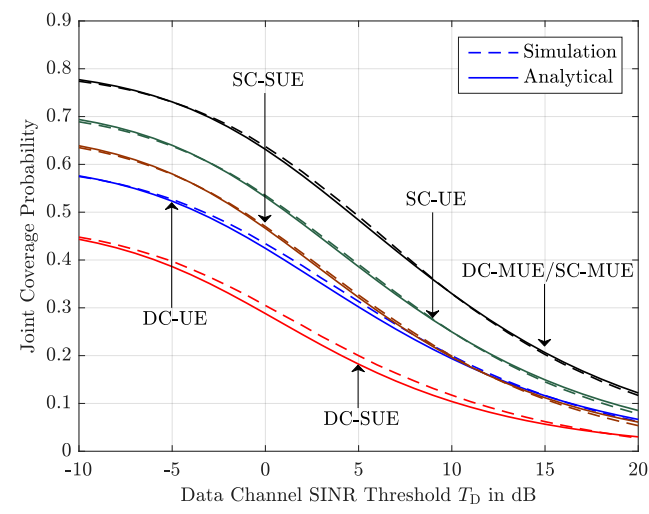

(a)

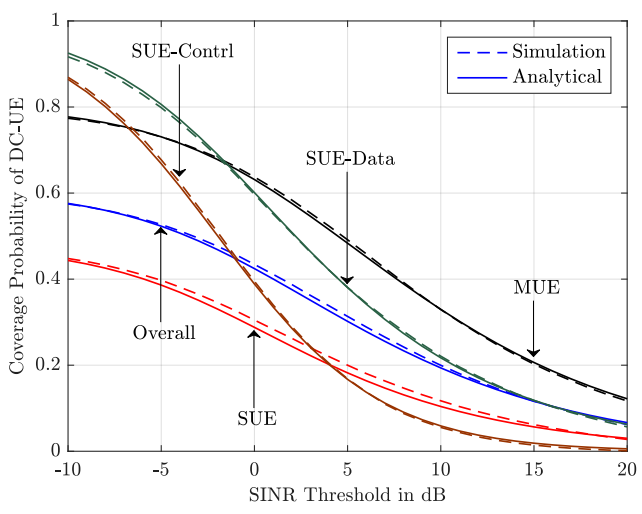

(b)

Fig. 4. Accuracy investigation when $\alpha_{2}=3.5, B_{2}=2$ and $\lambda_{2}=5 \lambda_{1}$ under multi-frequency tiering.

\subsection{Performance Analysis of Dual Connectivity vs. Single Connectivity}

Secondly, we compare the joint coverage performance of DC with traditional SC in Fig. 5.

It can be observed in Fig. 5(a) that SC-UE outperforms DC-UE in joint coverage performance. This degradation can be explained with the help of Fig. 4(a), which presents a same performance for SC-MUE and DC-MUE, but an obvious better performance for SC-SUE than DC-SUE. This is due to the fact that for DC-SUE, selecting SeNBs to transmit data signal means a shorter weighted distance between this UE and SeNB than MeNB, leading to a SINR decrease for signaling control message with MeNBs, while for DC-MUE, SC-MUE and SC-SUE, data channel and control channel both receive from the weighted nearest eNB, guaranteeing a relatively high SINR level. This comparison reveals that the increase in mobility robustness of DC is at the price of SINR degradation.

Fig. 5(b) details the control channel coverage performance for SC-SUE and DC-SUE. The result presents that increasing the density ratio of SeNBs to MeNBs will improve the control channel coverage performance of DC-SUE but will decrease that of SC-SUE. This is because that with denser SeNBs deployed in the network, some MUEs are likely to become SUEs, and the average link distance of the control channel is shortened for DC capable UE but stretched for DC incapable UE. For instance, suppose a DC-SUE $E_{0}$ and a DC-MUE ${ }_{1}$ receive control signals from the same MeNB, which also implies that DC-MUE ${ }_{1}$ lies closer than DC-SUE $\mathrm{E}_{0}$ to this MeNB with high possibility, providing DC-MUE ${ }_{1}$ with higher control channel SINR than DC-SUE ${ }_{0}$. When denser SeNBs are deployed with a new SeNB lies close to UE ${ }_{1}, D_{C}-M_{U} E_{1}$ may become DC-SUE ${ }_{1}$ by changing its data channel association from MeNB to SeNB. But due to DC mechanism, this new DC-SUE ${ }_{1}$ will maintain its control channel associated with the same MeNB, which still guarantees a higher SINR on the control channel than DC-SUE 0 , and hence improve the average control channel SINR for DC-SUEs. However, when it comes to UEs without DC capability, new SC-SUEs are quite possible to be located at the edge of newly deployed small cell, bringing in lower SINR on the control channel and hence decreasing the average control channel SINR for SC-SUEs. 


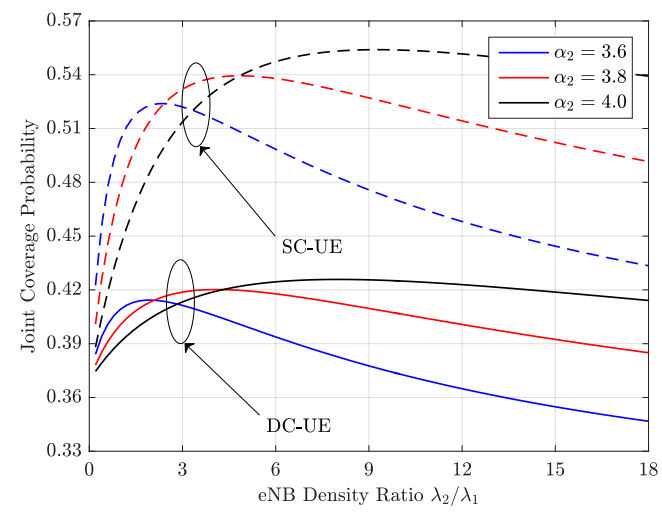

(a)

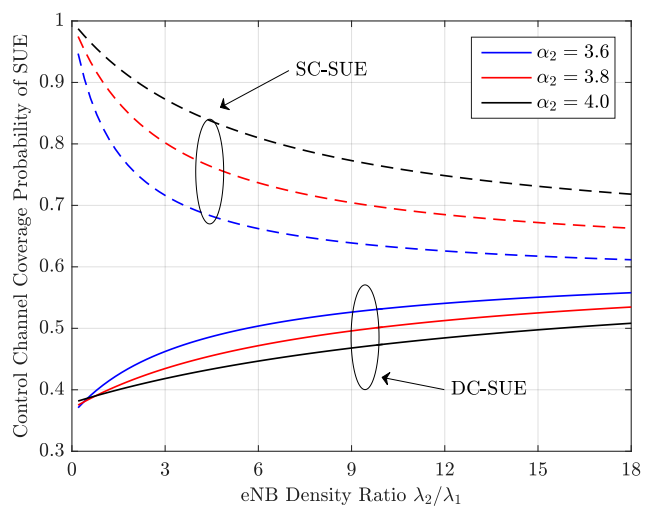

(b)

Fig. 5. Comparison between DC and SC when $T_{\mathrm{D}}=1 \mathrm{~dB}$ and $B_{2}=5$ under multi-frequency tiering.

\subsection{Performance Analysis of Multi-Frequency vs. Single-Frequency}

Then, we compare the impacts of different network tiering schemes on DC in Fig. 6.

The results in Fig. 6(a) reveals a frustrating trend for single-frequency tiering where the joint coverage probability performance monotically decreases with more SeNB deployed. This trend is in line with intuition, and is the result of the heavily increased aggregate interference from the newly deployed SeNBs. In single-frequency tiering, DC-UEs cannot benefit from denser SeNB deployment since adding more SeNBs will not shorten the link distance of DC-UE's control channel, which is always managed by the nearest MeNB. The severe performance degradation in control channel under single-frequency tiering is also proved in Fig. 6(b).

On the contrary, Fig. 6(a) reveals the existence of an optimal density ratio to achieve a maximum joint coverage probability under multi-frequency tiering. Under this tiering scheme, although Fig. 6(b) and Fig. 5(b) both illustrate an improved control channel coverage performance for DC-SUEs with the increase of SeNB density, the overall joint coverage performance of all DC-UEs does not present a monotically increasing trend, implying that DC-SUEs and DC-MUEs can be balanced to improve the overall joint coverage performance.

Besides the greater space for optimization, multi-frequency tiering also significantly outperforms single-frequency tiering in the joint coverage performance, which proves multi-frequency tiering a more feasible networking scheme for dual connectivity.

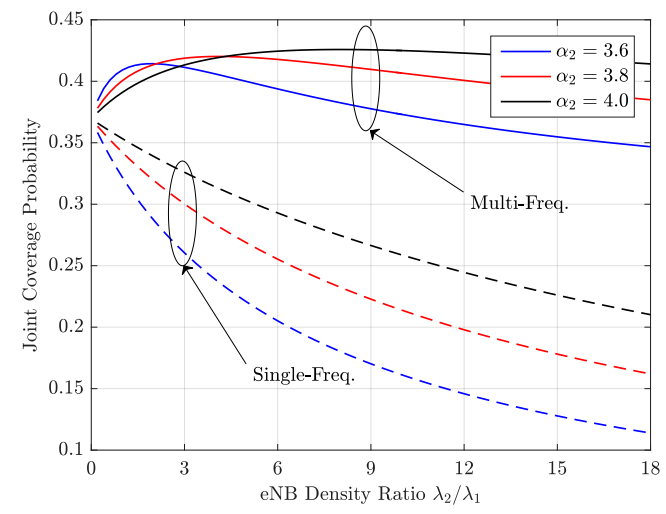

(a)

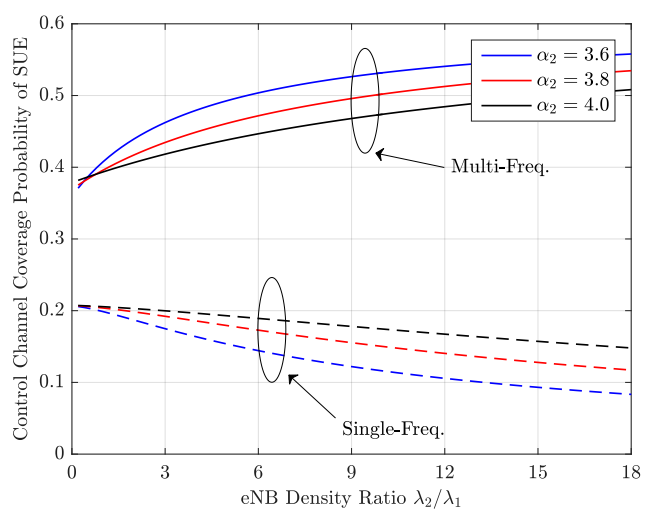

(b)

Fig. 6. Comparison between tiering schemes for DC-UE when $T_{\mathrm{D}}=1 \mathrm{~dB}$ and $B_{2}=5$. 


\subsection{Analysis of Path Loss Exponent and Association Bias}

Finally, we investigate the optimal density ratio and the impacts of different network parameters for DC under different tiering schemes in Fig. 7.

Fig. 7(a) shows the maximum joint coverage probabilities with various data channel association biases and path loss exponents under multi-frequency tiering. It is demonstrated that in greater signal attenuating environments (i.e., larger $\alpha_{2}$ ), higher maximum joint coverage probability can be expected with greater corresponding optimum density ratio. Another interesting observation is that this optimum density ratio will decrease when UEs are encouraged to associate their data channel with SeNBs (i.e., larger $B_{2}$ ), while the maximun joint coverage probability remains the same as the horizontal dashed line illustrated. To interprete this observation, Corollary 1 can be helpful, which shows the constraint between transmit power, association bias, and density ratio by presenting the joint coverage probability with $u$ in (22) and (23). This corollary reveals that eNB densities, transmit powers and association biases influence the function domain horizontally by spanning or squeezing the curve shape rather than the codomain of joint coverage probability. The achievable maximum value is fixed once the SINR targets are setup under a certain signal propagation scenario.

In Fig. 7(b), the joint coverage performance of DC under single-frequency tiering again proves frustrating, which provides a design guidance preventing direct utilization of DC in single-frequency tiered ultra dense HCN without efficient interference management.

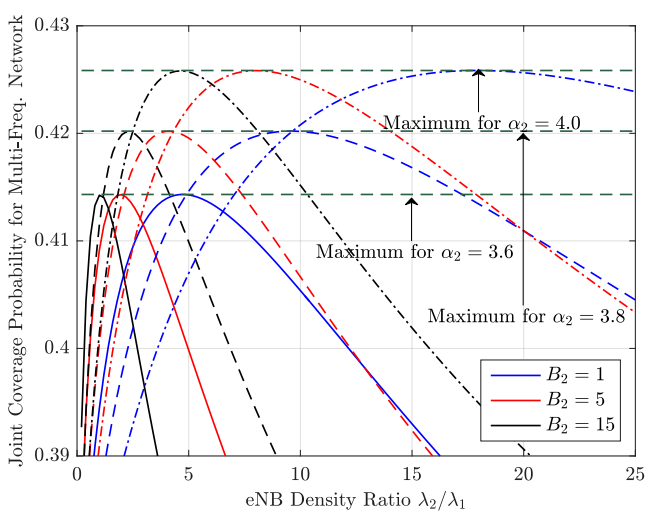

(a)

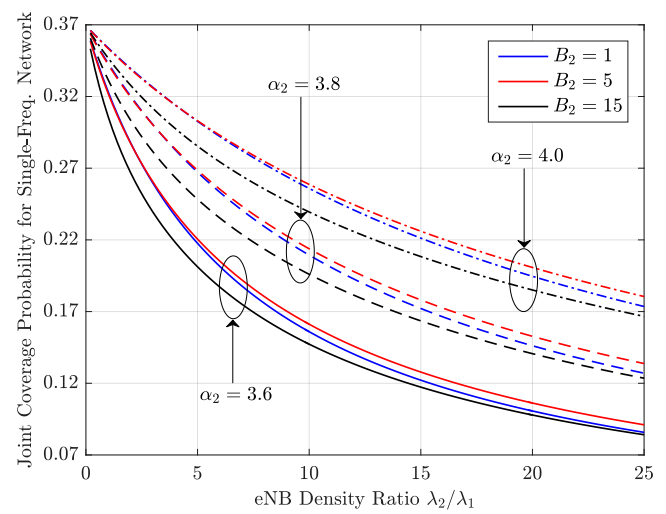

(b)

Fig. 7. Impacts of path loss exponent and association bias on DC-UE when $T_{\mathrm{D}}=1 \mathrm{~dB}$.

\section{Conclusion}

This paper presents a tractable framework for the downlink SINR analysis of DC assisted $\mathrm{HCN}$ under both multi-frequency and single-frequency tiering. Accurate expressions of data-control joint coverage probabilities are derived, allowing efficient further investigations to provide design insight for the utilization of DC. Numerical results reveal that although DC is beneficial in the mobility robustness of HCN, it also brings in SINR degradation for the control channel. Moreover, with more preferable joint coverage performance, multi-frequency tiering proves to be a more feasible networking scheme in the implementation of DC than single-frequency tiering. Besides, multi-frequency tiering also allows optimization through adjusting eNB density ratio to achieve the maximal joint coverage probability for DC, where different cell association biases only influence the value of the optimal density ratio not the value of this maximal joint coverage probability. 
The work in this paper also allows several future extensions. One possible extension is to design opportunistic mechanism to alleviate the SINR degradation on the control channel. Another extension is to design partial frequency reuse scheme to balance single-frequency tiering and multi-frequency tiering, which can provide a trade-off between joint coverage performance and spectral efficiency. Besides, tractable framework to quantify the benefit of DC in mobility robustness is also of interest, which should be a significant complement to the SINR analysis in this work.

\section{References}

[1] J. Andrews, "Seven ways that HetNets are a cellular paradigms shift," IEEE Communications Magazine, vol. 51, no. 3, pp. 136-144, Mar. 2013. Article (CrossRef Link).

[2] J. Zhang, X. Yang, Q. Yao, et al., "Cooperative Energy Efficiency Modeling and Performance Analysis in Co-Channel Interference Cellular Networks,” The Computer Journal, vol. 56, no. 8, pp. 1010-1019, Aug. 2013. Article (CrossRef Link).

[3] C. Yang, J. Li, Q. Ni, et al., "Interference-Aware Energy Efficiency Maximization in 5G Ultra-Dense Networks," IEEE Transactions on Communications, vol. 65, no. 2, pp. 728-739, Feb. 2017. Article (CrossRef Link).

[4] Y. Li, B. Cao and C. Wang, "Handover Schemes in Heterogeneous LTE Networks: Challenges and Opportunities,” IEEE Wireless Communications, vol. 23, no. 2, pp. 112-117, Feb. 2016. Article (CrossRef Link).

[5] M. Jo, T. Maksymyuk, R. Batista, et al., "A Survey of Converging Solutions for Heterogeneous Mobile Networks,” IEEE Wireless Communications, vol. 21, no. 6, pp. 54-62, Dec. 2014. Article (CrossRef Link).

[6] H. Zhang, X. Chu, W. Guo, et al., "Coexistence of Wi-Fi and Heterogeneous Small Cell Networks Sharing Unlicensed Spectrum,” IEEE Communications Magazine, vol. 53, no. 3, pp. 158-164, Mar. 2015. Article (CrossRef Link).

[7] C. Rosa, K. Pedersen, H. Wang, et al., "Dual connectivity for LTE small cell evolution: functionality and performance aspects," IEEE Communications Magazine, vol. 54, no. 6, pp. 137-143, Jun. 2016. Article (CrossRef Link).

[8] P. Legg, P. Fotiadis and P. Soldati, "Load balancing and aggregation algorithms for LTE dual connectivity,” in Proc. of IEEE Vehicular Technology Conference (VTC'2016 Spring), Nanjing, China, May 2016, pp. 1-5. Article (CrossRef Link).

[9] Y. Wu, Y. He, L. Qian, et al., "Traffic scheduling and power allocations for mobile data offloading via dual-connectivity," in Proc. of IEEE International Conference on Communications (ICC’2016), Kuala Lumpur, Malaysia, pp. 1-5, May 2016. Article (CrossRef Link).

[10] M. Pan, T. Lin, C. Chiu, et al., "Downlink traffic scheduling for LTE-A small cell networks with dual connectivity enhancement," IEEE Communications Letter, vol. 20, no. 4, pp. 796-799, Apr. 2016. Article (CrossRef Link).

[11] H. Wang, G. Pocovi, C. Rosa, et al., "Configuration of dual connectivity with flow control in a realistic urban scenario," in Proc. of IEEE Vehicular Technology Conference (VTC'2015 Fall), Boston, USA, pp. 1-5, Sep. 2015. Article (CrossRef Link).

[12] H. Wang, C. Rosa and K. Pedersen, "Inter-eNB flow control for heterogeneous networks with dual connectivity,” in Proc. of IEEE Vehicular Technology Conference (VTC'2015 Spring), Glasgow, Scotland, pp. 1-5, May 2015. Article (CrossRef Link).

[13] G. Pocovi, S. Barcos, H. Wang, et al., "Analysis of heterogeneous networks with dual connectivity in a realistic urban deployment," in Proc. of IEEE Vehicular Technology Conference (VTC'2015 Spring), Glasgow, Scotland, pp. 1-5, May 2015. Article (CrossRef Link).

[14] Z. Wu, W. Xie, F. Yang, et al., "User association in heterogeneous network with dual connectivity and constrained backhaul," China Communications, vol. 13, no. 2, pp. 11-20, Feb. 2016. Article (CrossRef Link). 
[15] A. Mukherjee, "Macro-small cell grouping in dual connectivity LTE-B networks with non-ideal backhaul,” in Proc. of IEEE International Conference on Communications (ICC'2014), Sydney, Australia, pp. 2520-2525, Jun. 2014. Article (CrossRef Link).

[16] A. Mukherjee, "Optimal flow bifurcation in networks with dual base station connectivity and non-ideal backhaul," in Proc. of Asilomar Conference on Signals, Systems and Computers (Asilomar'2014), Pacific Glove, USA, pp. 521-524, Nov. 2014. Article (CrossRef Link).

[17] S. Ahmad and D. Datla, "Distributed power allocations in heterogeneous networks with dual connectivity using backhaul state information,” IEEE Transactions on Wireless Communications, vol. 14, no. 8, pp. 4574-4581, Aug. 2015. Article (CrossRef Link).

[18] Y. Liu, H. Jiang, E. Tong, et al., "A MRO scheme for dual connectivity in LTE small cell enhancement scenario,” in Proc. of International Conference on Communications and Networking in China (ChinaCom'2015), Shanghai, China, pp. 1-5, Aug. 2015. Article (CrossRef Link).

[19] S. Barbera, L. Gimenez, L. Sánchez, et al., "Mobility sensitivity analysis for LTE-Advanced HetNet deployments with dual connectivity," in Proc. of IEEE Vehicular Technology Conference (VTC’2015 Spring), Glasgow, Scotland, pp. 1-5, May 2015. Article (CrossRef Link).

[20] L. Giménnez, P. Michaelsen and K. Pedersen, "Analysis of data interruption in an LTE highway scenario with dual connectivity,” in Proc. of IEEE Vehicular Technology Conference (VTC'2016 Spring), Nanjing, China, pp. 1-5, May 2016. Article (CrossRef Link).

[21] J. Andrews, F. Baccelli and R. Ganti, “A tractable approach to coverage and rate in cellular networks,” IEEE Transactions on Communications, vol. 59, no. 11, pp. 3122-3134, Nov. 2011. Article (CrossRef Link).

[22] H. Jo, Y. Sang, P. Xia, et al., "Heterogeneous cellular networks with flexible cell association: a comprehensive downlink SINR analysis,” IEEE Transactions on Wireless Communications, vol. 11, no. 10, pp. 3484-3495, Oct. 2012. Article (CrossRef Link).

[23] S. Singh, H. Dhillon and J. Andrews, "Offloading in heterogeneous networks: modeling, analysis, and design insights," IEEE Transactions on Wireless Communications, vol. 12, no. 5, pp. 2484-2497, May 2013. Article (CrossRef Link).

[24] Y. Yang, Z. Liu, B. Min, et al., "Optimal user density and power allocation for device-to-device communication underlaying cellular networks,” KSII Transactions on Internet and Information Systems, vol. 9, no. 2, pp. 483-503, Feb. 2015. Article (CrossRef Link).

[25] K. Yang, X. Zhang, S. Wang, et al., "Spectrum allocation and service control for energy saving based on large-scale user behavior constraints in heterogeneous networks," KSII Transactions on Internet and Information Systems, vol. 10, no. 8, pp. 3529-3550, Aug. 2016. Article (CrossRef Link).

[26] S. Chiu, D. Stoyan, W. Kendall, et al., Stochastic Geometry and its Applications, 3rd Edition, John Wiley \& Sons Ltd, 2013. Article (CrossRef Link). 

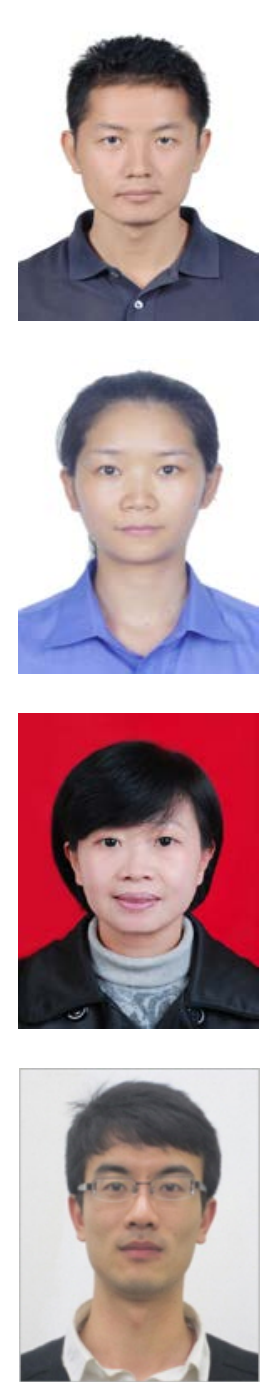

Xianling Wang received his Ph.D. degree in communication and information system from Beijing University of Posts and Telecommunications, Beijing, China, in 2014. He is currently working in Xiamen University of Technology. His research interests include multi-antenna transmission, LTE system, and stochastic geometry based wireless network modeling.

Min Xiao received her Ph.D. degree in communication and information system from Xiamen University, Xiamen, China, in 2011. She is currently an associate professor of Communication Engineering at Xiamen University of Technology. Her research interests include channel coding and network coding.

Hongyi Zhang received her Ph.D. degree in system structure of computer from Xidian University, Xi'an, China, in 2008. She is currently a professor of Computer Science at Xiamen University of Technology. Her research interests include intelligent information and signal processing.

Sida Song received his Ph.D. degree in communication and information systems from Beijing University of Posts and Telecommunications, Beijing, China, in 2015. He is currently working in Huawei Technologies Co., Ltd. His research interests include $5 \mathrm{G}$ wireless systems, statistical modeling of wireless networks, and stochastic geometry. 